\title{
Ubiquitin modifications
}

\author{
Kirby N Swatek ${ }^{1}$, David Komander ${ }^{1}$ \\ ${ }^{I}$ Medical Research Council Laboratory of Molecular Biology, Francis Crick Avenue, Cambridge, CB2 0QH, UK
}

Protein ubiquitination is a dynamic multifaceted post-translational modification involved in nearly all aspects of eukaryotic biology. Once attached to a substrate, the 76-amino acid protein ubiquitin is subjected to further modifications, creating a multitude of distinct signals with distinct cellular outcomes, referred to as the 'ubiquitin code'. Ubiquitin can be ubiquitinated on seven lysine (Lys) residues or on the N-terminus, leading to polyubiquitin chains that can encompass complex topologies. Alternatively or in addition, ubiquitin Lys residues can be modified by ubiquitin-like molecules (such as SUMO or NEDD8). Finally, ubiquitin can also be acetylated on Lys, or phosphorylated on Ser, Thr or Tyr residues, and each modification has the potential to dramatically alter the signaling outcome. While the number of distinctly modified ubiquitin species in cells is mind-boggling, much progress has been made to characterize the roles of distinct ubiquitin modifications, and many enzymes and receptors have been identified that create, recognize or remove these ubiquitin modifications. We here provide an overview of the various ubiquitin modifications present in cells, and highlight recent progress on ubiquitin chain biology. We then discuss the recent findings in the field of ubiquitin acetylation and phosphorylation, with a focus on Ser65-phosphorylation and its role in mitophagy and Parkin activation.

Keywords: ubiquitin; proteasomal degradation; phosphorylation; post-translational modification; Parkin Cell Research (2016) 26:399-422. doi:10.1038/cr.2016.39; published online 25 March 2016

\section{Introduction}

The realization by Goldknopf et al. that histones can be modified by the protein ubiquitin through Lys-linked isopeptide bonds [1] marked a new era in post-translational signaling. At the time, small chemical modifications of amino acids, including phosphorylation and acetylation, were already known to regulate protein function $[2,3]$. The prevalence and importance of protein-based modifications emerged in the 1980 s, when landmark studies connected the ATP-dependent ubiquitination of substrates to their degradation by the $26 \mathrm{~S}$ proteasome [4]. 35 years later, it is clear that this function of ubiquitin was just the tip of an enormous iceberg.

The post-genomic era provided insight into the complexity of the ubiquitin system, and far over 1000 proteins regulate ubiquitination in human cells [5]. Ubiquitin is attached to substrates by a sophisticated three-step enzymatic cascade [6], utilizing E1 ubiquitin activating [7], E2 ubiquitin conjugating [8] and a variety of E3 ubiquitin ligating enzymes [9-11]. Ubiquitinated proteins are

Correspondence: David Komander

E-mail: dk@mrc-lmb.cam.ac.uk recognized by receptors that contain ubiquitin-binding domains (UBDs) [12], and several specialized families of proteases, the deubiquitinases (DUBs), remove ubiquitin modifications $[13,14]$. Comprehensive proteomics studies identified tens-of-thousands of ubiquitination sites on thousands of proteins [15-17]. It appears that most proteins will experience ubiquitination at some point in their cellular lifetime.

Ubiquitination starts by the attachment of a single ubiquitin molecule to a substrate Lys residue. These monoubiquitination events are abundant [18] and have many roles in cells [19]. This review focuses on the multitude of signals that can be generated when the attached monoubiquitin is modified further. We have previously referred to this complexity in the ubiquitin system as the 'ubiquitin code' [19], borrowing nomenclature from the histone modification field. The 'histone code' describes the complex interplay of modifications that histones are subjected to, and for which many rules of interdependent, hierarchical assembly and disassembly have been functionally dissected [20]. As will be discussed below, the understanding of the ubiquitin code has advanced rapidly in recent years, however, new methods and insights are required to comprehensibly crack this complex code of post-translational modifications. 


\section{Ubiquitin modifications - a conceptual overview}

Ubiquitin is a 76-amino acid protein, and as such bears many potential sites for additional post-translational modifications. The key features of ubiquitin are its seven Lys residues, all of which can be ubiquitinated, to give rise to isopeptide-linked ubiquitin chains. An eighth chain type, Metl-linked or 'linear' chains, is generated when ubiquitin is attached to the N-terminus of a second ubiquitin (Figure 1A). Proteomics studies show that all possible linkage types co-exist in cells $[16,17,21$ 23]. Lys48-linked chains are the predominant linkage type in cells (often $>50 \%$ of all linkages, see references above), and their role is to target proteins to the proteasome for degradation [4]. In contrast, the second most abundant chain type linked via Lys63 performs various non-degradative roles [24]. More recently, research has begun to characterize the remaining, 'atypical' ubiquitin modifications (linked through Met1, Lys6, Lys11, Lys27, Lys29 or Lys33). Surprisingly, this has led to the discovery of highly linkage-specific enzymes and proteins that assemble, recognize and hydrolyze each ubiquitin chain type, i.e., 'write', 'read' and 'erase' this first layer of the ubiquitin code. On the basis of previous reviews on this topic $[19,25]$, the first part of this review gives an update to the exciting developments in ubiquitin chain signaling, providing a conceptual framework for future discoveries.

New layers of the ubiquitin code are currently emerg- ing, based on findings that ubiquitin can not only be ubiquitinated, but also be modified by other modifications. These include modifications by the small ubiquitin-like (Ubl) modifier (SUMO) family [26-28]. SUMOylation of (poly)ubiquitin is conceptually similar to polyubiquitin chain formation. It is less clear whether other Ubl modifiers are attached to ubiquitin under physiological conditions.

More strikingly, ubiquitin is also modified by small chemically distinct post-translational modifications such as phosphorylation and acetylation. Mining of available datasets indicates that 6 out of 7 ubiquitin Lys residues can become acetylated [29-32] (Figure 1B). Ubiquitin phosphorylation on Ser57 has been identified in some of the earliest ubiquitin proteomics experiments [15]. Subsequent studies confirmed this finding and further revealed a broad array of phosphorylation sites scattered across the surface of ubiquitin (Figure 1C). These sites include Thr7, Thr12 [33], Thr14 [34], Ser20 [35], Thr22, Thr55, Thr66 [36], Tyr59 [37], and Ser65 [38]. This cornucopia of modifications on ubiquitin has a vast potential to provide additional regulation in the ubiquitin system.

Despite their existence, the kinases and acetyltransferases ('writers'), phosphatases and deacetylases ('erasers') and phospho-ubiquitin (phosphoUb) or acetyl-ubiquitin (AcUb) binding domains ('readers') for these modifications are mostly unknown, with few exceptions. The second part of this review highlights these new find-
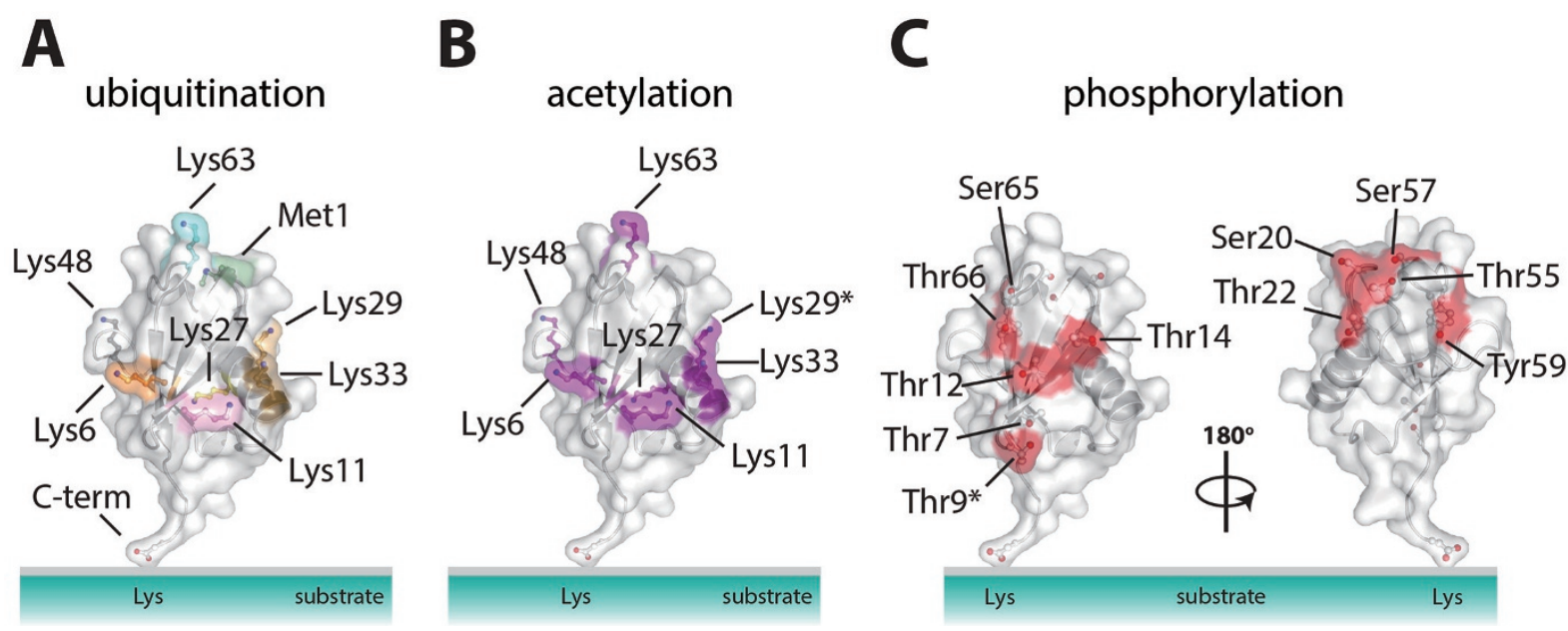

Figure 1 Modification sites on ubiquitin. Ubiquitin is shown as a cartoon under a semitransparent surface, and modifiable residues are shown in ball-and-stick representation with blue nitrogen and red oxygen atoms. (A) Structure of ubiquitin highlighting the eight sites of ubiquitination. (B) Six out of seven Lys residues on ubiquitin have been reported to be acetylated in proteomics datasets. An asterisk marks the seventh Lys, Lys29, for which acetylation has not been identified to date. (C) Identified phosphorylation sites of ubiquitin are displayed according to proteomic analysis. Red spheres indicate phosphorylatable hydroxyl groups on Ser/Thr and Tyr residues. The structure was rotated 180 degrees to show all phosphorylation sites. An asterisk on Thr9 indicates this site is ambiguously assigned. 
A
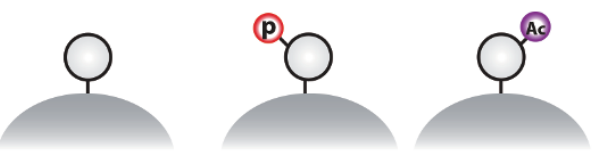

monoUb
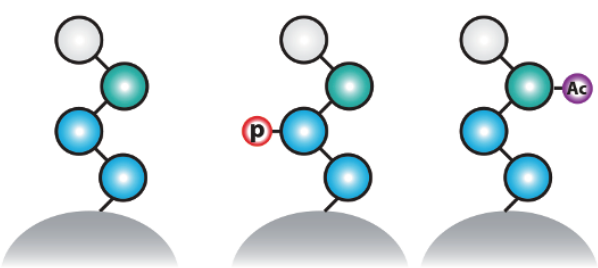

modified mixed

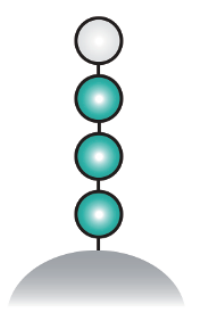

homotypic
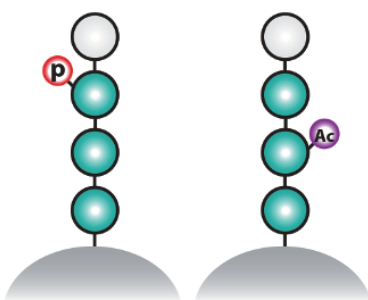

modified homotypic

\section{mixed}
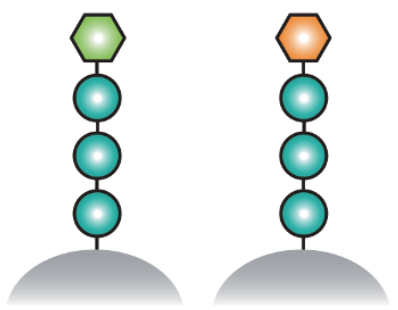

Ubl-modified Ub
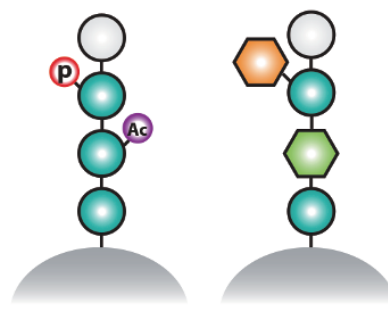

mixing modifications

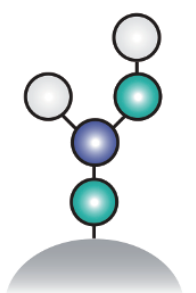

branched
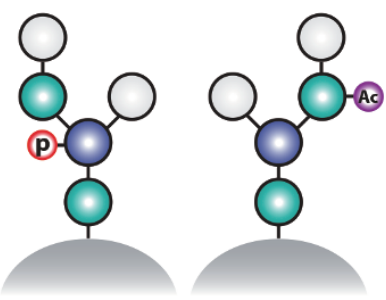

modified branched

B
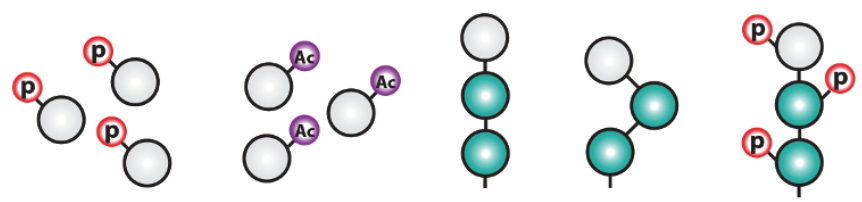

second messengers

Figure 2 New complexity in the ubiquitin code. (A) Conceptual representation of some of the possible ubiquitin-, Ubl (NEDD8, SUMO2/3)- and chemical modifications of ubiquitin. (B) Unanchored ubiquitin and ubiquitin chains, with or without modifications, can function as second messengers in cells.

ings concerning these modifications, and focuses on Ser65-phosphoUb, and its roles in mitophagy and Parkin activation.

\section{A significantly expanded code}

The new layers of ubiquitin modifications significantly alter the features of the ubiquitin code as previously described [19]. Monoubiquitination of proteins can occur with straight or modified ubiquitin, and chains can be homotypic (one linkage type) or heterotypic (Figure 2A). In the latter, chains of one linkage type can be extended by a second type, forming a non-branched structure. Alternatively, a ubiquitin molecule in a chain may be ubiquitinated at multiple Lys residues, forming a 'branched' (also known as 'forked') structure. At this point, we have to assume that all of the ubiquitin moieties, even in complex topologies, could be modified further by acetylation or phosphorylation, or both (Figure 2A).

Considering eight linkage types between ubiquitin 
molecules, alternative modifications of ubiquitin Lys residues with Ubls or acetyl-groups, and eleven potential phosphorylation sites, this generates an essentially unlimited number of potential combinations.

The different ubiquitin signals can be attached to substrates, and invoke a substrate-specific response. In addition, 'unanchored' ubiquitin or ubiquitin chains exist in cells, and perform second-messenger-like functions [39-41]. Monoubiquitin is a prominent component of cell lysates, and once phosphorylated or acetylated, could act as new signaling molecules, in a second-messenger-like fashion (Figure 2B). Indeed, 'free' Ser65-phosphoUb can be detected in cells [42], and may activate kinase signaling [43] (see below). Moreover, unanchored polyubiquitin may be DUB-resistant once phosphorylated [44, 45] (see below), and perform functions as a comparably stable independent signaling entity.

\section{Studying ubiquitination events}

At this point, it is important to briefly discuss the available tools to understand ubiquitin modifications, which have become quite sophisticated in recent years (Figure 3). New biochemical methods exploit linkage-specific DUBs [46] and UBDs [47, 48]. Linkage-specific antibodies to Met1-, Lys11-, Lys48- and Lys63-linked chains as well as against Ser65-phosphoUb have been developed and provided highly useful reagents [42, 49-51]. Nevertheless, mass spectrometry has had the most profound impact on studying the ubiquitin code [52]. Quantitative techniques using cell (SILAC) and peptide (TMT) labeling as well as absolute quantification employing labeled ubiquitin peptide standards (AQUA) can be used in conjunction with antibodies enriching ubiquitinated peptides, and enabled many insights into the ubiquitin system. The role of mass spectrometry in studying ubiquitination has been reviewed in-depth recently [52].

It is important to realize, though, that mass spectrometry-based methods depend on tryptic digestion of ubiquitin and in-depth analysis of the resulting ubiquitin peptides. While this enables annotation and quantification of relative and absolute amounts of certain modifications with the right tools and workflows, it prevents an in-depth understanding of the interplay between modifications. For example, a new area of research dealing with 'branched' ubiquitin chains, in which one ubiquitin molecule is ubiquitinated at multiple sites, is severely compromised by the inability to study all endogenous branched chains. Moreover, it appears quite likely that certain ubiquitin phosphorylation events only exist in the context of particular polyubiquitin chains. It is this

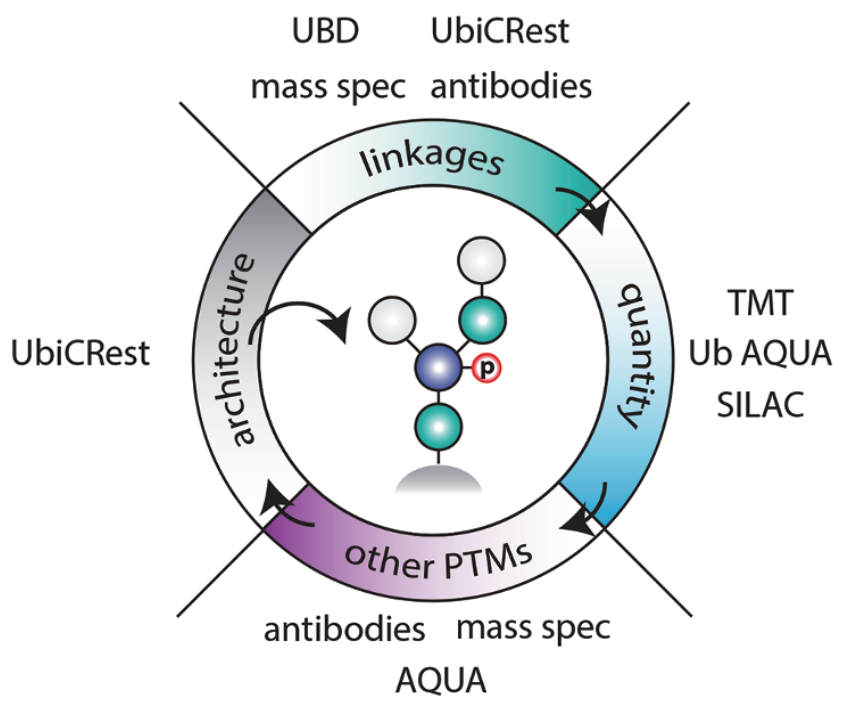

Figure 3 Studying Ub modifications. Linkage-specific UBDs [47, 48], Ubiquitin Chain Restriction (UbiCRest) analysis [46], linkage-specific antibodies [49-51], a Ser65-phosphoUb antibody [42] and mass spectrometry [52] allow for the identification of chain types and ubiquitin modifications. Mass spectrometry enables the quantitation of all ubiquitin linkages in a sample. Relative quantitative techniques include tandem mass tag (TMT) labeling and stable isotope labeling by amino acids in cell culture (SILAC), while absolute quantification (AQUA) strategy determines the exact quantities of each ubiquitin chain type [52]. Similar strategies are available to identify and quantify small molecule and Ubl modifications of ubiquitin.

information about the hierarchy in modifications that has advanced the histone field, and new methods and tools are essential to achieve a similar degree of insights into the ubiquitin code.

\section{New insights into ubiquitin chain signaling}

Nonetheless, the available methodologies and tools have provided exciting new insights into ubiquitin signaling, and much progress has been made on the enzymatic machinery of the ubiquitin system and functions of differently linked polyubiquitin signals. We previously reviewed the roles of atypical ubiquitin chains [19, 25], however this field has continued to advance rapidly. Below, we provide an update and discuss recent developments for all chain types, and insights into ubiquitin chain architecture. Strikingly, recent data have shaken long-standing dogmas about what constitutes a minimal proteasomal targeting signal, and based on this, we discuss a 'ubiquitin threshold' model for proteasomal degradation. 
A

E2 or E3
(exemplary) Parkin UBE2S RNF168 UBE3C AREL1 UBE2R1 NEDD4L LUBAC

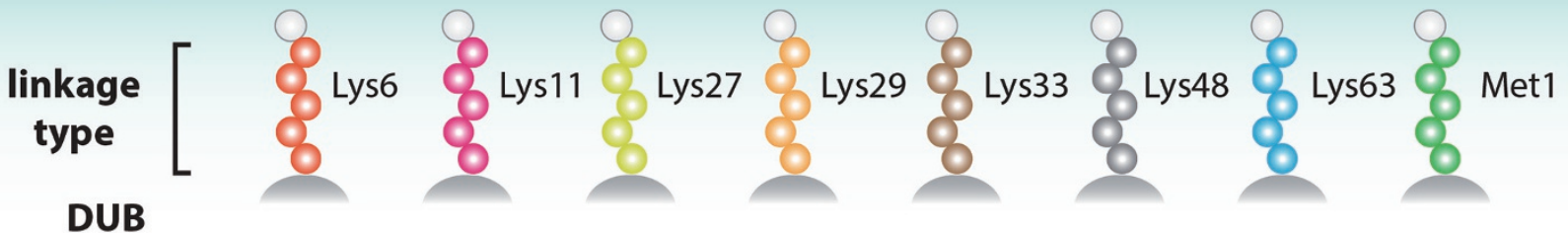
(exemplary) USP30
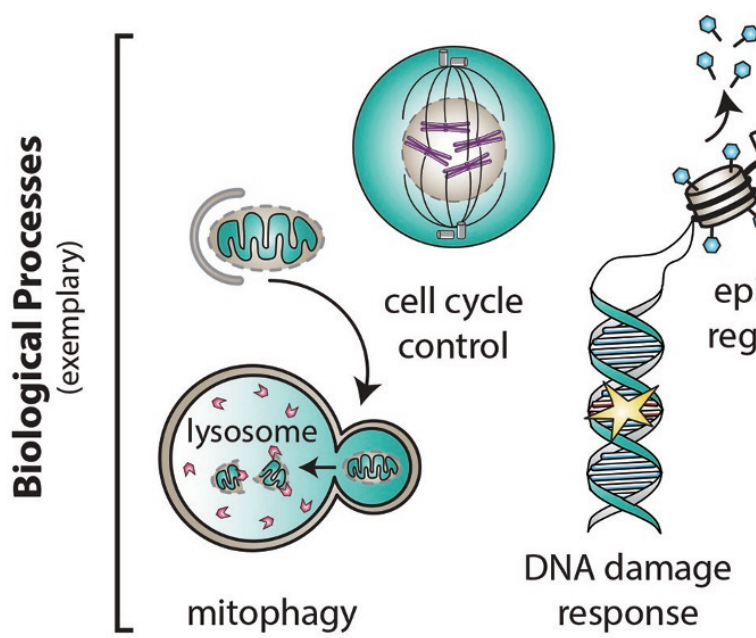

TRABID TRABID

OTUB1

AMSH OTULIN

B
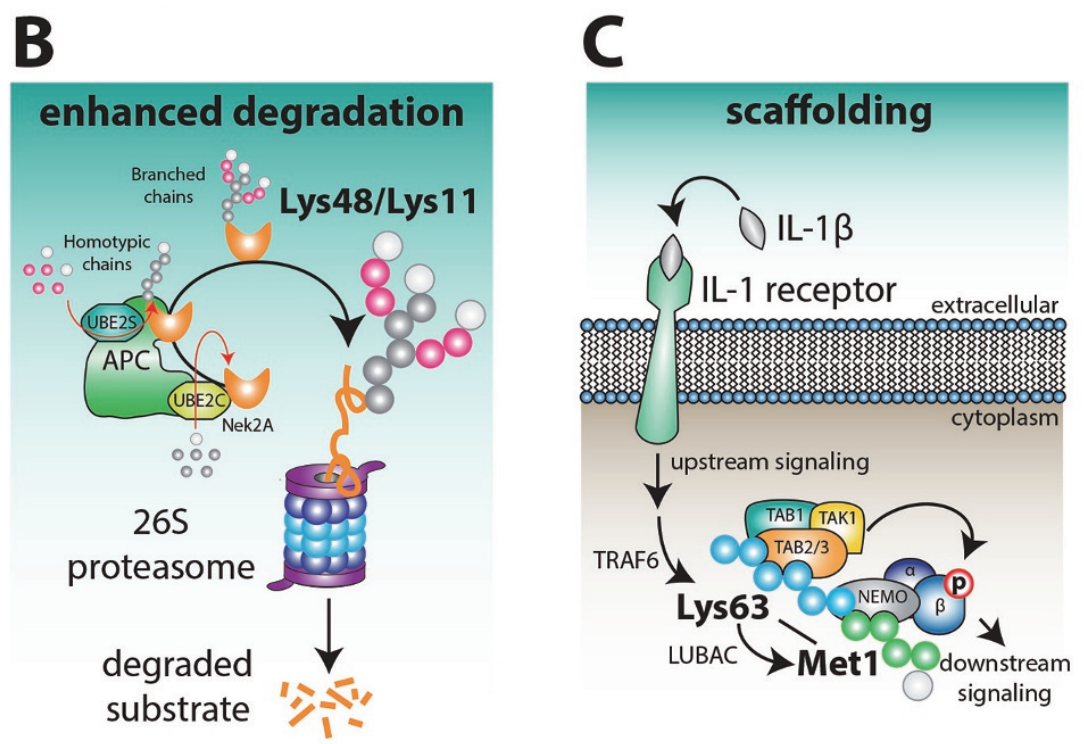
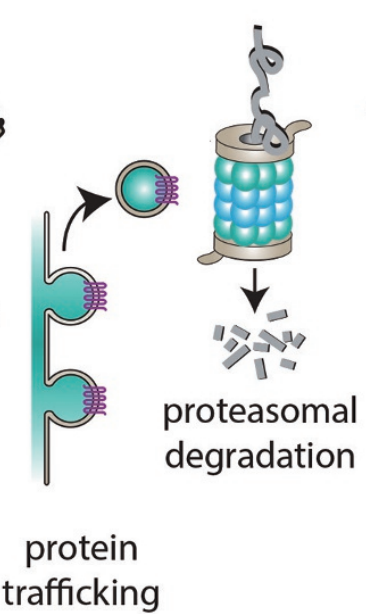

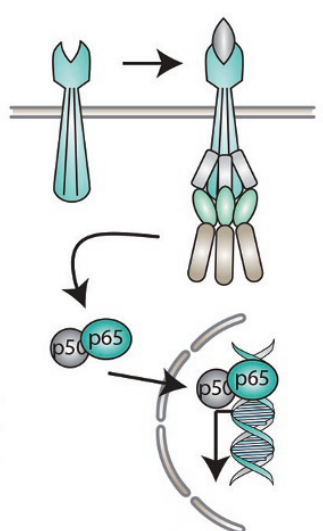

NFKB signalling

Figure 4 Physiological roles associated with individual chain types. (A) A small selection of E2 or E3 enzymes that assemble and DUBs that disassemble ubiquitin chains with linkage preferences is indicated. Below, cartoons illustrate some of the (new) biological processes that particular linkage types have been linked with as discussed in the text. (B) APC/C is active during early mitosis and modifies cell cycle regulators such as Nek2A with Lys48/Lys11-linked branched polyubiquitin. In this process, UBE2C first assembles short chains on the substrates, and these are then elongated on each ubiquitin by Lys11-linked polymers. Lys48/Lys11 branched chains enhance proteasomal degradation. (C) Mixed or branched Lys63/Met1-linked chains serve as protein scaffolds at immune receptors, such as IL-1 receptors, to promote NF- $\mathrm{B}$ signaling. (D) A viral E3 ligase initiates endocytic internalization of the MHC class I receptor through the attachment of mixed or branched Lys11/Lys63-linked ubiquitin chains. 


\section{Met1-linked 'linear' ubiquitin chains}

Research on Met1-linked 'linear' ubiquitin chains exemplifies how studies of a particular chain type can advance biology. The field started with the identification of the linear ubiquitin chain assembly complex (LUBAC), which includes an E3 ligase of the RING-between-RING (RBR) family, HOIP, that assembles exclusively Met1linked polyubiquitin [53] (Figure 4A). Subsequently, the identification of the Met1-linkage specific UBAN (ubiquitin binding in ABIN and NEMO) domain in NEMO (a component of the Inhibitor-of- $\kappa \mathrm{B}$ kinase (IKK) complex), linked Met1-linked chains to NF- $\mathrm{B}$ activation $[54,55]$. Indeed, LUBAC regulates signaling of tumor necrosis factor (TNF) and related cytokines [56, 57], and Met1-linked chains were found attached to components of the large complexes assembled at their receptors, such as NEMO and RIPK1 $[56,58]$. Indeed, we now understand the molecular basis of LUBAC specificity in exquisite biochemical and structural detail [59-62], as well as Met1-linkage specific ubiquitin binding by several small UBDs [55, 63-65]. In addition, a plethora of genetic models have confirmed important roles of LUBAC in inflammation and immunity [58, 66-72], and LUBAC components are also mutated or ablated in human inflammatory conditions $[73,74]$. The physiological roles of Met1-linked ubiquitin chains have been reviewed extensively $[75,76]$.

Initially, it was unclear which DUB would antagonize Met1-linked polyubiquitin. The ubiquitin-specific protease (USP) enzyme CYLD cleaves Lys63- and Met1linked polyubiquitin chains with similar activity [54, 77]. More strikingly, complete Met1-linkage specificity could be revealed in OTULIN (ovarian tumor (OTU) domain DUB with linear linkage specificity, also known as FAM105B or Gumby) [78, 79] (Figure 4A). OTULIN uses a mechanism of ubiquitin-assisted catalysis, in which Met1-linked polyubiquitin directly regulates the catalytic center of the enzyme [78]. Consistent with the role of Met1-linked chains, OTULIN could regulate NF$\kappa \mathrm{B}$ signaling [78, 80-82], but interestingly, OTULIN also affects Wnt signaling [79, 83]. A role for Met1-linked chains in this pathway has not been described, and requires further studies. Mechanistically, both CYLD and OTULIN regulate Met1-linked chains by directly interacting with LUBAC, both binding mutually exclusive to the N-terminal PUB domain of the catalytic subunit HOIP [81-84]. Another OTU DUB, A20, inhibits NF$\kappa \mathrm{B}$ signaling via a distinct route. A20 binds Met1-linked chains via a C-terminal 'A20-like $\mathrm{ZnF}$ ' domain $[64,65]$, but its N-terminal OTU domain is unable to hydrolyze this linkage type [85], and the relevance of A20's DUB function has been questioned [86]. Recent work suggests that upon activation by phosphorylation, A20 is able to hydrolyze Lys63-based scaffolds in cytokine signaling complexes, to which it is recruited via recognition of Met1-linked chains [87]. The interplay between DUBs, and their roles in Met1-linkage ubiquitin and NF- $\mathrm{KB}$ signaling are a subject of intense study.

Taken together, Met1-linked ubiquitin chains are now established to be key positive regulators of NF- $\mathrm{KB}$ signaling (Figure 4A). Much of the progress made in this area has been facilitated by the identification and characterization of the cellular machineries that assemble, bind and hydrolyze Met1-linked polyubiquitin. This approach serves as a model for research into other, less-well studied, chain types.

\section{(Re)emerging roles for Lys6-linked polyubiquitin}

In contrast to Met1-linked polyubiquitin, physiological roles of Lys6-linked chains are less clear. Lys6-linked chains are not enriched upon proteasome inhibition [16], potentially indicating non-degradative roles. Global quantitative proteomics studies recently associated this linkage type with two cellular contexts. One study showed that Lys6- as well as Lys33-linkages are upregulated upon UV genotoxic stress [88]. This is interesting since earlier findings concerning this linkage type associated it with the BRCA1/BARD1 E3 ligase complex, an important regulator of the DNA damage response [89, 90]. Tools for specific detection of Lys6-linked chains are still missing, and it will be interesting to study the localization and consequences of this modification after DNA damage.

A second set of studies has linked Lys6-linked chains to mitochondrial homeostasis [91-93]. Mitophagy, which is described in greater detail in the second part of this review, requires ubiquitination of damaged mitochondria, which is mediated in large part by the E3 ligase Parkin. Like HOIP, Parkin belongs to the RBR family, and assembles predominantly Lys6- and Lys11-, as well as smaller amounts of Lys48- and Lys63-linked chains in vitro [91, 92] and on depolarized mitochondria [91, 93] (Figure 4A). Using a ubiquitin replacement strategy or overexpression of ubiquitin mutants, it was shown that mutations of Lys6 and Lys63 (but not Lys48 or Lys11) delayed mitophagy, suggesting that these linkage types invoke specific downstream processes [93, 94].

An intriguing Lys6 preference has also been identified in the DUBs regulating mitophagy and Parkin. USP30, the only DUB constitutively localized to mitochondria, counteracts mitophagy $[93,95,96]$ and has a preference for Lys6-linked polyubiquitin [44, 93] (Figure 4A). This is noteworthy, USP family DUBs are typically non-specific [97] (with CYLD being another exception), and 
the molecular basis for this specificity is unclear. DUBs regulating mitophagy are discussed in more detail in the second part of this review.

Finally, roles of Lys6-linked chains may emerge in other processes such as xenophagy, since Parkin restricts intracellular mycobacteria [98]. Interesting in this context is that a bacterial effector E3 ligase, NleL, from enterohaemorrhagic Escherichia coli (EHEC), also assembles Lys6-linkages [99, 100]. Its pathophysiological function is unclear, but it is remarkable that bacteria appear to exploit the entirety of the ubiquitin code.

\section{Lys11-linked chains in the cell cycle and beyond}

Together with Met1-linked chains, Lys11-linked chains are the best studied of the atypical chain types, and have been established as an additional proteasomal degradation signal used in particular in cell cycle regulation [101, 102]. The Anaphase promoting complex/ cyclosome $(\mathrm{APC} / \mathrm{C})$ utilizes the only known Lys11-specific E2 enzyme, UBE2S, to assemble this chain type on substrates (Figure 4A) [102] . Whilst originally regarded as an independent degradation signal, recent data showed that substrates modified with homotypic Lys11-linked chains are poor substrates for the proteasome [103]. Indeed, the predominant form of APC/C-mediated ubiquitination is in the form of branched conjugates, which bind better to the proteasome and are more efficient in promoting protein degradation [103-105] (Figure 4B).

Clear roles for homotypic Lys11-linked polyubiquitin have not been established, however, two DUBs from the OTU family exhibit striking specificity for this linkage type, namely Cezanne/OTUD7B and Cezanne2/OTUD7A (Figure 4A) [85]. Reported physiological roles for Cezanne include regulation of non-canonical NF- $\kappa \mathrm{B}$ signaling [106], T-cell activation [107], and EGF receptor trafficking [108], but a clear link to Lys11-linked chains has not been established in these studies.

Cezanne also regulates the adaptation of cells to low oxygen conditions, by regulating hypoxia-inducible factors, HIF- $1 \alpha$ and HIF- $2 \alpha[109,110]$. Cezanne knockdown leads to increased Lys11-linked ubiquitination of the transcription factor HIF- $1 \alpha$, and to its subsequent degradation, but this effect could not be rescued by proteasome inhibition. Instead, degradation of HIF-1 $\alpha$ in this context likely involves chaperone-mediated autophagy [109]. Cezanne also regulates HIF-2 $\alpha$, in this case through a transcriptional mechanism. HIF- $2 \alpha$ is regulated by the transcription factor E2F1, and the loss of HIF- $2 \alpha$ caused by Cezanne knockdown can be rescued by overexpression of E2F1 [110]. E2F1 degradation may be regulated by a cell cycle and APC/C-dependent mechanism, however, E2F1 loss caused by Cezanne knockdown also could not be rescued by proteasome inhibitors [110].
Despite these emerging roles, it is striking that receptors for Lys11-linked polyubiquitin have remained obscure. Identification of linkage-specific UBDs will likely fuel our understanding of this chain type, and may reveal new functional context.

\section{The enigma: Lys27-linkages}

Of all the 'atypical' ubiquitin chains, Lys27-linked chains remain the least-well understood and studied. Initial data showing them as a chain type assembled by Parkin [111] have remained unconfirmed, and a linkage-specific DUB or UBD has proved elusive (Figure 4A).

Two E3 ligases have recently been suggested to assemble Lys27-linked chains in cells. A RING E3 ligase, RNF168, was reported to assemble Lys27-linked ubiquitin chains on histones H2A and H2A.X in cells [112] (Figure 4A). RNF168 is mechanistically interesting as it is one of the few RING E3 ligases with additional UBDs that may bind and orient an 'acceptor' ubiquitin and potentially furnish the ligase with linkage selectivity. Consistent with known roles for RNF168, Lys27-linked chains were upregulated by a DNA damaging reagent that induce DNA double-strand breaks, and shown to recruit DNA damage repair factors, such as p53 binding protein 1 (53BP1), to DNA damage foci. Together, these data imply that Lys27-linked chains may serve as scaffolds for protein recruitment in the DNA damage response (DDR) (Figure 4A).

A second E3 ligase suggested to assemble Lys27linked chains is HECT domain and ankryin repeat containing E3 ubiquitin ligase 1 (HACE1). Using ubiquitin mutants, two papers independently reported that HACE1 modifies its substrates Optineurin (OPTN, see below) and Y-box binding protein 1 (YB-1), with Lys27-linked polyubiquitin $[113,114]$. The reported roles for Lys27linked modification in these instances are regulation of protein secretion (YB-1) and increase of autophagic flux (OPTN).

All three reports suggest that Lys27-linked chains act to recruit proteins, suggesting the presence of specific UBDs, and indeed, UBDs from DDR components interact well with Lys27-linked diubiquitin [112]. With these interesting leads, Lys27-linked chains are likely to become less enigmatic in the near future.

New roles for Lys29-linked chains in proteasome regulation and epigenetics

For Lys29- and Lys33-linked chains the first set of linkage-specific proteins for assembly, recognition and hydrolysis is now known, enabling genetic approaches and detailed studies into chain function. Lys29-linked chains are assembled by the HECT E3 ligase KIAA10/ UBE3C that assembles K29- and K48-linked polyubiq- 
uitin chains [115-117] (Figure 4A), and associates with the $26 \mathrm{~S}$ proteasome $[115,118]$. Interestingly, while the yeast orthologue Hul5 also resides on proteasomes, it assembles Lys63- rather than Lys29-linked chains [119]. In human cell lines, proteasome inhibition results in enrichment of Lys29-linked chains [16], suggesting that Lys29linked chains can be a proteasomal degradation signal. However, UBE3C modifies the proteasome ubiquitin receptor Rpn13 with Lys29-linked chains in response to proteasomal stress, to prevent further substrate engagement [118]. This could be an alternative reason why this chain type is enriched in proteomics studies with inhibited proteasomes.

Beyond proteasomal degradation, new insights into Lys29-linkages emerged from the identification of Lys29/ Lys33-specificity in the deubiquitinase TRABID (Figure 4A). TRABID contains an extended linkage-specific OTU domain $[120,121]$, and in addition, an N-terminal UBD module with three Npl4-like zinc finger (NZF) domains that recruit TRABID to Lys29/Lys33-linked polyubiquitin chains in cells $[116,117,121]$. Initial data on TRABID linked it to Wnt-mediated transcription [122], and a recently generated mouse knockout model identified its roles in epigenetic regulation [123] (Figure 4A). In this model, Trabid regulates transcription of a set of interleukins (IL12 and IL23) downstream of Tolllike receptor (TLR) signaling, yet in an indirect way. It was reported that a target of Trabid in this pathway is the histone demethylase Jmjd2d, which it appears to deubiquitinate and stabilize, so that Jmjd2d can act on the interleukin gene promoters to release repression [123]. Indeed, loss of Trabid leads to modification of Jmjd2d with Lys29- and Lys11-linked chains, linking these chain types to functional outcome.

\section{Lys33-linked polyubiquitin in intracellular trafficking}

The HECT E3 ligase AREL1 was shown to make Lys33- as well as Lys11-linked polyubiquitin chains, and was used to generate Lys33-linked chains in large quantities for the first time [116, 124] (Figure 4A). AREL1 has been scarcely studied. In a single report, a role as a negative regulator of apoptosis was suggested, whereby AREL1 may ubiquitinate antagonists of inhibitor of apoptosis (IAP) proteins, including SMAC, HtrA2 and ARTS [125]. The modification is suggested to lead to protein degradation. However, chain type(s) involved in cells were not studied; in vitro reconstitution assays showed predominantly Lys33-linkages on the three substrates as a result of AREL1-catalyzed ubiquitination [116].

Lys33-linked chains have been implicated in various biological processes [25], and more recently, in post-Gol- gi membrane protein trafficking [126] (Figure 4A). The BTB domain-containing adapter protein, KLHL20, works with a Cullin-3 E3 ligase complex to catalyze Lys33-linked polyubiquitination of Coronin 7(Crn7), which promotes its recruitment to the trans-Golgi network (TGN), indicating roles in anterograde trafficking [126]. These roles for Lys33-linked chains were revealed using an elegant ubiquitin replacement strategy, in which cellular ubiquitin is replaced with a K33R ubiquitin mutant. However, similar to much of the other research on atypical chains, most insights into Lys33-linkages are based on isolated studies and require follow-up research to solidify specific roles.

\section{Lys48-linked chains - re-defining a proteasomal degra- dation signal}

As mentioned above, Lys48-linked chains are the most common chain type and target proteins for proteasomal degradation (Figure 4A). Elegant biochemical studies by the Pickart lab showed that proteins require at least a tetraubiquitin chain to be efficiently targeted to the proteasome [127]. This tetraubiquitin dependence was the dogma in the field for the last decade, despite subsequent structures of the proteasome lid that illuminated its ubiquitin chain receptors and DUBs, and did not quite explain the tetraubiquitin requirement [128-130]. Moreover, the ubiquitination pattern of efficiently degraded proteins such as Cyclin B1 revealed multiple short chains of various linkages, rather than singular long chain [131]. Also, the models did not explain how some proteins can use single Lys48-linked chains for non-degradative means [132], although protection by UBDs likely plays a role [133].

Interesting biochemical and biophysical studies have started to re-define the minimal requirements for proteasomal degradation [134]. In this system, a substrate modified with four monoubiquitin modifications was not degraded. Interestingly, two diubiquitin modifications were a better degradation signal as compared to a single tetraubiquitin chain [134]. More detailed studies along these lines, controlling for chain type, chain length and chain position on a substrate, appear essential to finally understand what constitutes the perfect proteasomal degradation signal. Differences in these three parameters could easily lead to distinct degradation kinetics, and a new layer of regulation, as suggested in the initial study [134].

Lys63-linked chains and the fight for the crown in inflammatory signaling

The second 'canonical' Lys63-linked chain type has many well-studied (and extensively reviewed) non-deg- 
radative roles [19, 24, 25]. Most notoriously, this linkage type was at the heart of a debate in inflammatory signaling and NF- $\kappa \mathrm{B}$ activation, that started when Met1-linked chains were found to be important in the underlying signaling cascades (Figure 4A). Specific UBDs for each chain type are present in the cascading kinase complexes - the 'upstream' TAK1 kinase complex contains Lys63-linkage specific UBDs in TAB2 and TAB3 [135137], while the 'downstream' IKK complex encodes Met1-linkage specificity in the NEMO UBAN domain $[54,55,138]$ (see above).

The debate has been elegantly resolved by biochemical findings, which clearly show that Lys63-linked chains are modified with Met1-linked chains in mixed or branched architectures [139] (Figure 4C). LUBAC binds to polyubiquitin of various compositions [57], and the mechanism of HOIP requires binding of an acceptor ubiquitin that could be part of an existing chain [61]. Heterotypic or 'hybrid' chains were revealed by using linkage-specific DUBs in a method called 'ubiquitin chain restriction analysis' [46], where a Lys63-linkage specific DUB released blocks of Met1-linked polyubiquitin, and a Met1-linkage specific DUB removed predominantly the high-molecular weight portion of the polyubiquitin smear [139], showing that the chains started with Lys63-linkages on the substrate.

Mixed-linkage Lys63/Met1-linked chains seem like the solution to recruit distinct kinase complexes, and may resolve a debate about the specificity of NEMO (Figure 4C). In addition to its UBAN domain, NEMO contains a zinc-finger UBD module that was reported to prefer Lys63-linkages [140, 141]. Two UBDs with distinct specificities may indeed target the IKK complex to Lys63/Met1 linkage junctions within the polymer.

\section{The rise of 'invisible' branched and mixed chains}

As exemplified by Lys63/Met1 hybrid chains, mixed and branched chains could be charged with new signaling information. Indeed, all the above-mentioned new roles for atypical chains may rely on interplay with other linkage types. Jmjd2d ubiquitination involves Lys11and Lys29-linked chains [123], and HECT and RBR E3 ligases often assemble a defined subset of linkages (NleL, Lys6/Lys48 [99]; HACE1, Lys27/Lys48 [113]; UBE3C, Lys29/Lys48 [115]; AREL1, Lys33/Lys11 [116]). Consistently, when enriched from cells, Lys29-linked chains are predominantly part of heterotypic polyubiquitin [117].

The power of branched chains to provide new complexity to the system is highlighted by research on the APC/C, which assembles Lys48/Lys11-branches that are efficient proteasomal degradation signals [103-105] (Figure 4B), or viral E3 ligases that assemble branched chains to initiate endocytosis of host proteins [142, 143] (Figure 4D). In contrast, earlier reports indicated that some types of branched chains lead to non-degradable ubiquitin structures and proteasome stalling [144], and are actively prevented in cells [145]. We think that both possibilities are likely, and much of the outcome will depend on how the (proteasomal) DUBs can handle complex chain architectures.

Additional roles for chain branching are likely to be identified in the coming years. However, as also described above, we are essentially blind to these modifications, as the methods to distinguish chains generally do not report on chain architecture. Mass spectrometry can only cleanly identify those branches in which two neighboring Lys residues are modified, such as Lys29/Lys33 [15] or Lys6/Lys11 [144]. The majority of branched conjugates - there are 28 different combinations in which one ubiquitin can be modified on two Lys or Met1 residues - cannot be identified or quantified easily. Current methods rely on limited proteolysis by trypsin and hence suffer from systematic errors [146, 147]. It is also not clear, whether higher-order branched ubiquitin (ubiquitin ubiquitinated on more than two Lys residues) exists in cells. To study this complexity in chain architecture, new methods need to be developed that enable analysis of the branched ubiquitin pool of a cell.

\section{A 'ubiquitination threshold' model for proteasomal degradation}

The new insights into individual chain types, new rules for proteasomal degradation, and rise of branched and mixed linkage chains, support a 'ubiquitination threshold' model for proteasomal degradation (Figure 5). Clearly, the main task of the ubiquitin system is proteasomal degradation, and the proteasome can degrade a range of substrates in various contexts, not all of which depend on (poly)ubiquitination [148]. With the new data on degradation signals (see section on Lys48-linkages above) the number of distinct signals to initiate degradation increases further; multiple modifications with short Lys48-linked chains, or branched structures with Lys 11- or Lys48-linkages are clearly efficient signals. In other words, a protein performing a ubiquitin-mediated, non-degradative task (e.g., modified with a Lys33linked chain), once modified on another nearby substrate Lys, or modified on the ubiquitin chain itself to create a branched structure, could with little effort be destined for proteasomal degradation. Indeed, the possibility of branching would enable any non-degradative chain type to become a degradation signal. The transition from defined degradation signals (Lys48-linked tetraubiquitin) 


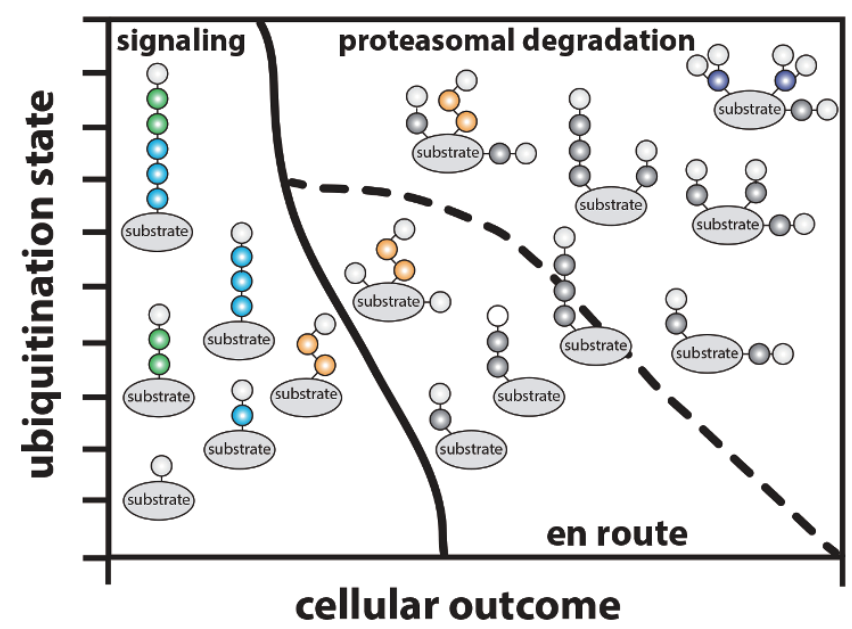

Figure 5 A 'ubiquitin threshold' model for proteasomal degradation. Substrate ubiquitination can result in two general outcomes, cellular signaling or proteasomal degradation. Recent evidence supports a model in which multiple short chains (e.g., diubiquitins) or branched ubiquitin are better degradation signals as compared to a single Lys48-linked tetraubiquitin. These findings also suggest that non-degradative ubiquitin signals could be modified into degradative signals through addition of short and/or branched ubiquitin chains to substrates.

to a 'ubiquitination threshold' model, where the amount of polyubiquitin rather than the type is important, may explain a lot of existing biochemical and proteomic data. For example, the most abundant E2 enzymes such as those of the UBE2D family, appear to modify proteins on random sites with short chains of many types [144], and Cyclin B degradation is facilitated by multiple short chains of various types [131].

In contrast, specialized degradative E3 ligase systems such as SCF E3 ligases, utilize the Lys48-specific E2 enzyme UBE2R1, which assembles chains of medium-lengths (on average 3-6 ubiquitin molecules) on model substrates in vitro [149] to serve as efficient 'canonical' degradation signals (Figure 4A).

Another important component in this context is the fact that ubiquitination is highly dynamic. DUBs regulate which chain types are assembled, and how the ubiquitination status of a protein will be shaped. Many E3 ligases exist in conjunction with DUBs, and could perform essential chain editing functions [13]. Interesting in this context is also the idea that the length and dynamics of ubiquitin chains could determine their susceptibility to DUBs and determine the relative stability of a ubiquitin signal $[150,151]$. To conclusively discuss what defines the 'perfect' proteasomal degradation signal, we need to be able to study chain length and chain branching in more detail.

Conceptually, the idea that 'signaling' pools of ubiquitinated proteins could be very similar to 'proteasome-targeted' pools, provides challenges to studying the roles of the signaling chain types. It is the former, likely very small pool of proteins, that will reveal the signaling roles of atypical chains. Especially for the studies of lowly abundant atypical chains, the search for these 'needles-in-the-haystack' will continue to be challenging, but as discussed above, the 'magnets' to extract them and uncover their context are becoming stronger.

\section{Alternative ubiquitin modifications: a second layer in ubiquitin signaling}

So far, we have discussed ubiquitin chains, and the many forms and functions that this first layer of the ubiquitin code entails. A second layer is provided when ubiquitin is modified with other kinds of modifications, which could include conjugation by other Ubl modifiers, or small chemical modifications such as phosphorylation or acetylation. The remainder of the review will discuss these modifications, which present new complexity, yet also new functionality and ways to regulate the ubiquitin system.

\section{SUMOylated and NEDDylated (poly)ubiquitin}

SUMOs constitute the best studied Ubl modification system, which competes with ubiquitination for modifications of Lys residues. As compared to the ubiquitin system, the relative simplicity of the SUMO system with a single E1, a single E2 and a small number of E3 enzymes has enabled a comprehensive set of mechanistic studies [152]. Physiological roles of SUMOylation have emerged in many biological processes, in particular in transcription, DNA repair, and various stress responses [153]. Interestingly, SUMO also forms chains, and SUMO-targeted ubiquitin ligases ('StUbLs') target SUMO chains for ubiquitination [154].

In addition to ubiquitinated SUMO chains, and more relevant to this review, is the recent findings by proteomics studies that ubiquitin can be SUMOylated. Multiple ubiquitin Lys residues (Lys6, Lys11, Lys27, Lys48, and Lys63) can be targeted for SUMOylation, implying a potentially complex web of intertwining modifications [26-28]. While the functional roles of these SUMO-ubiquitin chain types are still ambiguous, SUMOylation of ubiquitin Lys6 and Lys27 is upregulated in response to heat shock and proteasome inhibition [28], providing a starting point for further investigation. Mechanistically, ubiquitin SUMOylation is also interesting. Typical 
SUMOylation motifs, such as a SUMO E2 binding site or a SUMO-interacting motif [153], are missing in the well-folded ubiquitin molecule, and hence, a ubiquitin-targeted SUMO ligase ('UbtSL') should be responsible, but remains to be identified.

It is possible that other Ubl modifiers, such as NEDD8 or ISG15, also modify ubiquitin and ubiquitin chains. With overexpression of NEDD8, NEDDylated ubiquitin can be readily observed [155], however, overexpression leads to the non-physiological use of NEDD8 by the ubiquitination machinery [156]. It is unclear to what extent NEDD8 modifies ubiquitin under physiological conditions.

The idea that ubiquitin and polyubiquitin could be modified by Ubl modifications adds significant complexity to the system, and highlights the crosstalk between modifications (Figure 2).

\section{Ubiquitin phosphorylation and acetylation}

The modifications of amino acids by small chemical groups such as phosphate, methyl and acetyl groups, sugars or lipids are highly abundant and regulate many fundamental processes in biology. Most comprehensively studied are protein phosphorylation and acetylation, and their characterization was fueled by peptide-level affinity enrichment strategies that led to discoveries of tens-ofthousands of modified sites in proteomes [157]. Due to the wealth of proteomics datasets, it is not uncommon to find your protein of interest to be phosphorylated or acetylated or both. The common challenge, however, is to understand whether a modification is a genuine way to alter a protein's function, or whether it is a side reaction without relevance. Most available proteomics datasets provide steady-state snapshots without information on relative abundance (which is commonly $<1 \%$ of a protein pool), and often without dynamic information (i.e., changes of a modification upon a stimulus or over time etc.), making assessment of the 'importance' of particular modifications difficult.

It should not be a surprise that phosphorylation and acetylation extend to ubiquitin and Ubl modifiers, and while this information has been available for some time, the first studies into their physiological relevance have only surfaced in 2014. The identification of functionally important phosphorylation of ubiquitin at Ser65, performed by PTEN-induced protein kinase 1 (PINK1) during mitophagy (discussed below), has triggered enormous excitement and research into this and other chemical modifications of ubiquitin. Importantly, both types of modifications, Lys acetylation and Ser/Thr/Tyr phosphorylation, affect charge and surface properties of the ubiquitin molecule - particularly important since most surfaces of ubiquitin are functional in some contexts of the ubiquitination machinery or during ubiquitin binding. Below, we review what is known about ubiquitin acetylation and phosphorylation, as well as future directions and open questions.

\section{Codes collide with acetylated ubiquitin}

Lys acetylation competes with ubiquitin chain formation and hence impacts chain architecture. AcUb with modifications on Lys6 or Lys48 is readily detected in cells [29], but acetylation also occurs on other Lys residues (Figure 1B). Acetylated ubiquitin is readily available via non-natural amino acid incorporation, and this was used to assess the impact of AcUb on the ubiquitination machinery $[31,158]$. Lys6-AcUb and Lys48AcUb did not interfere with E1-mediated E2 charging, however, the discharge of ubiquitin onto substrates was inhibited in a variety of E2/E3 assembly reactions [31].

Importantly, the impact of Lys acetylation may not only extend to the modified Lys, but may also affect chain assembly or disassembly at nearby Lys residues. An intriguing example is the E2 enzyme UBE2S, which utilizes Lys6 in assembly of Lys11-linked polyubiquitin chains $[159,160]$ or the ubiquitination of MHC class I with Lys11/Lys63 branched chains, which also requires a functional Lys6 residue [142]. Hence, the effects of ubiquitin Lys acetylation may be manifold.

As discussed above, histones in nucleosomes are amongst the most modified proteins in eukaryotes in terms of density and variety of post-translational modifications [20]. Importantly, Lys6- and Lys48-AcUb has been identified attached to histones in cells [31]. An 'acetyl-mimetic' ubiquitin mutation (K6Q) was found to stabilize monoubiquitinated histone H2B [31]. Further research needs to address whether and how AcUb extends the histone code. It is tempting to speculate that the histone acetylation and deacetylation machineries also serve to modify the nearby attached ubiquitin molecules - after all, histones are the most abundantly ubiquitinated proteins in our cells.

Despite this emerging context, the enzymes responsible for ubiquitin acetylation and deacetylation, as well as functional role(s) of acetylated ubiquitin, are unknown. Recombinantly produced site-specific AcUb [31, 158] should enable identification of deacetylases, and may help to reveal AcUb-binding domains. Yet, as for other ubiquitin modifications, it is paramount to identify the acetyl-transferases that mediate ubiquitin acetylation to place AcUb into its cellular context. 
A

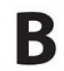

C

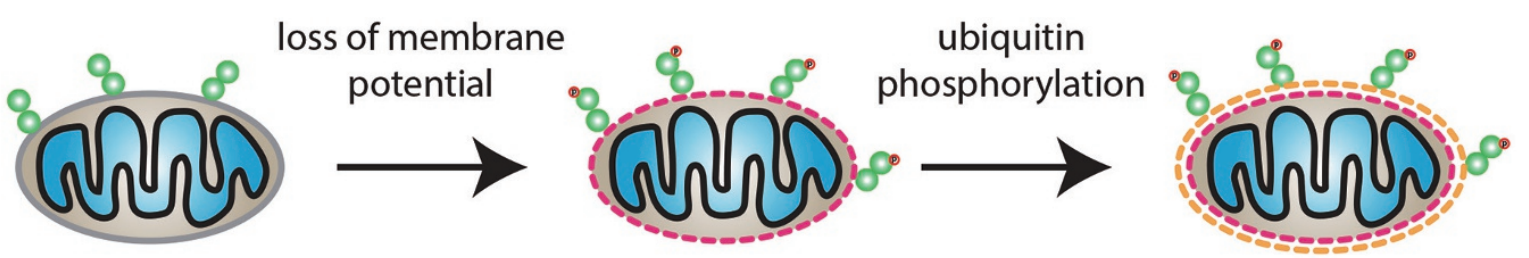

Homeostasis

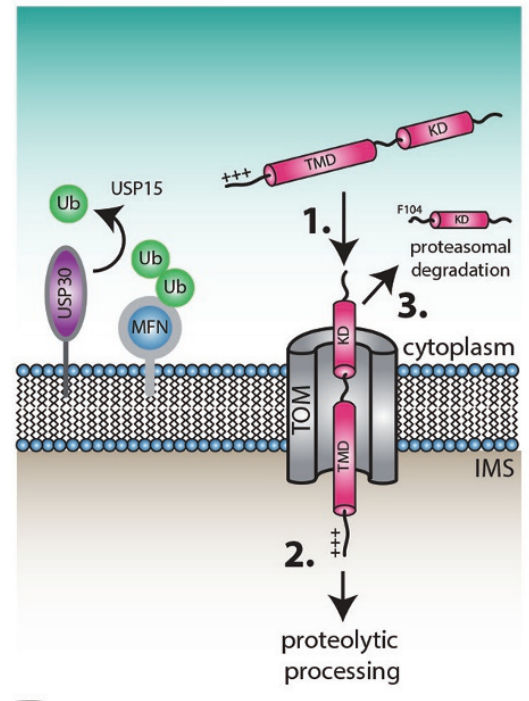

PINK1 stabilization

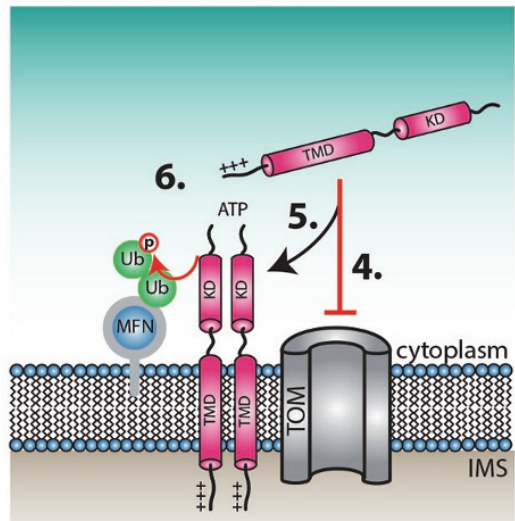

E
Parkin recruitment

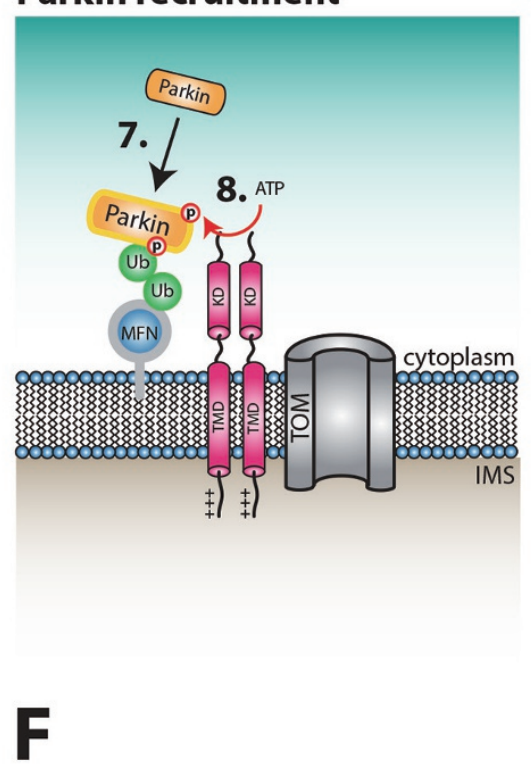

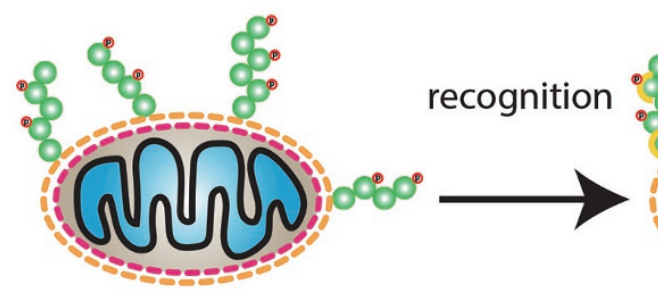

Parkin activation

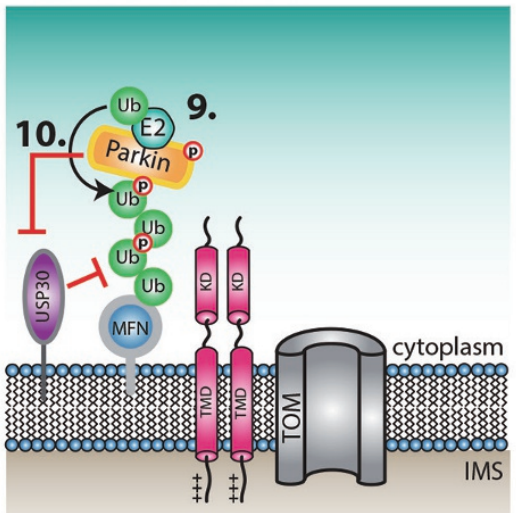

Receptor recognition

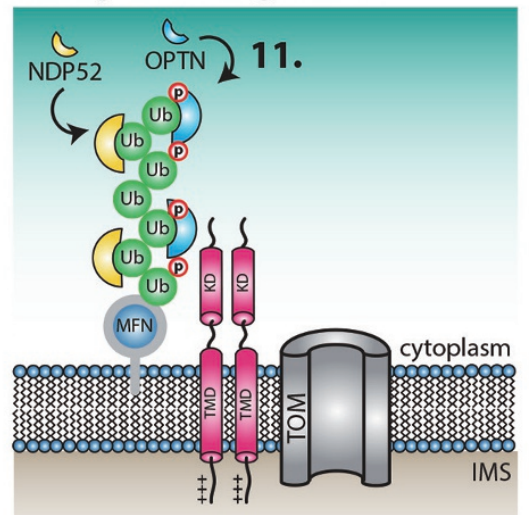

Autophagosome forms

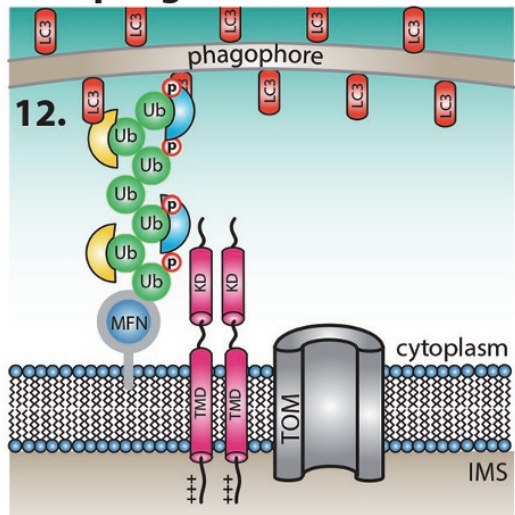


Increased complexity with phosphoUb, and links to Parkinson's disease

As with acetylation, evidence of ubiquitin phosphorylation on eleven sites was available in proteomics databases (Figure 1). Amongst these, Ser65-phosphorylation of ubiquitin is lowly abundant under steady state conditions, but dramatically increased when mitochondria are depolarized, providing cellular context of its regulation. These findings have initiated the emerging research field of ubiquitin phosphorylation. Whether and which kinases phosphorylate ubiquitin site-specifically is still unclear, however, the work on the PINK1/Parkin system exemplifies how such discoveries can come at a rapid pace.

In a nutshell, ubiquitin was identified as the main substrate for the protein kinase PINK1, which has been intensely studied due to its genetic links to autosomal recessive juvenile Parkinsonism (AR-JP), an early onset form of Parkinson's disease (PD). The discovery of ubiquitin phosphorylation originated from (a) the genetic link of PINK1 and Parkin, an E3 ligase also mutated in ARJP, (b) cell biological studies that placed PINK1 upstream of Parkin in mitophagy, (c) the search for the PINK1 substrate that acts as Parkin receptor on mitochondria and (d) the search for a mechanism of Parkin activation. This and the subsequent discoveries surrounding Ser65 phosphorylation are described below.

\section{Mitophagy - a new playground in ubiquitin re- search}

Mitophagy is the process by which cells selectively destroy damaged mitochondria in an autophagy-like fashion [161] (Figure 6). Mitochondria constitute a dynamic organelle network within cells, and are in constant flux with ongoing fusion and fission events. When parts of mitochondria are damaged, e.g., through accumulation of misfolded proteins or loss of membrane potential, the damaged parts of the mitochondria are tagged, isolated and subsequently destroyed [161]. A variety of mechanisms for destruction are in place, including autophagosomal degradation (mitophagy), but also proteasomal degradation and smaller-scale lysosomal degradation via vesicular routes [162]. Mitophagy has predominantly been studied by global depolarization of all cellular mitochondria, using uncoupling agents such as CCCP. Based on cell biological work, the mitochondrial protein kinase PINK1 was shown to be a key player in the process. PINK1 is constitutively degraded through proteolytic cleavage of its transmembrane domain by the transmembrane protease PARL [163], and subsequent ubiquitination and proteasomal degradation through N-end-rule E3 ligases [164] (Figure 6A). Upon mitochondrial damage, such as loss of membrane potential, PARL-dependent cleavage of PINK1 is inhibited and PINK1 accumulates on damaged mitochondria [165] (Figure 6B). PINK1 stabilization and catalytic activity lead to rapid accumulation of the E3 ligase Parkin on the outer mitochondrial surface [166] (Figure 6C). Many PINK1 substrates and Parkin receptors have been proposed [167, 168], but an important breakthrough came with the realization that PINK1 targets Parkin itself, phosphorylating it on Ser65 in the N-terminal Ubl domain $[169,170]$ (Figure 6C).

Seminal studies then showed that PINK1 also phosphorylates ubiquitin at Ser65 [44, 91, 171-173] (Figure 6C). Ubiquitin and the Parkin Ubl domain are highly similar in structure and both contain Ser65 in a structurally identical position $[172,173]$. In addition, mass spectrometry experiments comparing wild-type and PINK1knockout cells $[91,171]$ or analyzing reconstituted Parkin reactions [44] revealed a substantial increase for the Ser65-ubiquitin phosphorylation site only. Ser65-phosphoUb is barely detectable in cells lacking PINK1, and $<0.1 \%$ of total ubiquitin in wild-type cells without mitochondrial depolarization, but rises to more than $2 \%$ of the total ubiquitin pool in cells after CCCP-induced depolarization $[91,172]$. On mitochondria, $\sim 20 \%$ of all ubiquitins are phosphorylated at Ser65 after CCCP

Figure 6 Ser65-phosphorylation of ubiquitin in mitophagy signaling. (A) Under normal growth conditions, the TIM/TOM complex continually imports PINK1 into mitochondria (step 1). Upon entry, PINK1 undergoes proteolytic processing by the protease PARL (step 2) and is exported and degraded by the N-end rule pathway (step 3). USP30 controls the basal levels of mitochondrial ubiquitination. (B) Loss of mitochondrial membrane potential inhibits PINK1 import and proteolytic cleavage (step 4), leading to insertion of its transmembrane domain into the outer mitochondrial membrane (OMM) (step 5). PINK1 phosphorylates ubiquitin on mitochondrial proteins such as mitofusins (step 6). (C) Ser65-phosphoUb recruits Parkin to damaged mitochondria (step 7), and Ser65-phosphoUb binding releases the Parkin Ubl domain and enables its phosphorylation by PINK1 (step 8). (D) Ser65-phosphoUb binding and phosphorylation activate Parkin which subsequently ubiquitinates OMM proteins, and the newly incorporated ubiquitins are further phosphorylated by PINK1 (step 9). Parkin-mediated ubiquitination of USP30 facilitates its proteasomal degradation (step 10). (E) Mitophagy receptors NDP52 and OPTN bind to ubiquitinated mitochondrial proteins via their UBDs (step 11). (F) NDP52 and OPTN recruit the autophagy machinery to mitochondria (step 12). The phagophore engulfs mitochondria and fuses with the lysosome to degrade and recycle its contents. 
treatment $[91,94]$. The phosphorylated ubiquitin acts as a recruitment platform both for Parkin [91, 94, 171-175] (Figure 6D), and for autophagy adaptors that initiate mitophagy [176, 177] (Figure 6E and 6F). Discovery of PINK1 as the first ubiquitin kinase enabled not only wide-ranging studies into cell biology, but also into ubiquitin biochemistry and structure.

\section{Impact of ubiquitin phosphorylation on ubiquitin structure and enzymology}

Recombinant PINK1 enables access to Ser65-phosphoUb for biochemical and structural studies [178]. Surprisingly, Ser65 phosphorylation changes ubiquitin structure in novel ways, generating two ubiquitin conformations that are in dynamic equilibrium [44] (Figure 7A). The predominant 'major' conformation resembles the common ubiquitin structure that has been observed more than 300 times in the Protein Data Bank (PDB) [179, 180], but has important altered surface potential due to the phosphorylation on Ser65. A second, 'minor' conformation of ubiquitin had not been observed previously. In this conformation, the last $\beta$-strand and $\mathrm{C}$-terminal tail of ubiquitin are withdrawn into the molecule, altering many surface properties including the Ile44 patch and restricting the C-terminus for chain assembly [44] (Figure 7A). The relevance of the new conformation is not clear yet, but it seems likely that unknown binding partners could stabilize and recognize it specifically.

Moreover, striking effects of Ser65-phosphoUb on the ubiquitin system have been revealed (Figure 7B).
Ser65-phosphoUb is charged normally onto E2s by the E1 enzyme, but discharge into polyubiquitin chains is inhibited in several systems, most notably the UBE2N/ UBE2V1-mediated generation of Lys63-linked chains [44, 45]. Moreover, some RING E3 ligases, such as TRAF6, no longer assemble polyubiquitin chains with the UBE2D-family enzyme if only Ser65-phosphoUb is provided in the reaction [45]. Free Ser65-phosphoUb only accounts for a small percentage of the total ubiquitin pool though, and an impact on E3 ligase function seems unlikely.

However, interesting data suggest the existence of Ser65-phosphoUb-dependent protein kinase(s) [43]. In a search for new PINK1-dependent phosphorylation events, small GTPases of the Rab family were discovered as targets for phosphorylation, however, the protein kinase for Rab proteins was not PINK1 [43]. It is a likely scenario that in this instance, Ser65-phosphoUb, either on mitochondria or perhaps in its free form (second messenger role, Figure 2B), acts to activate a protein kinase for Rab proteins. The Parkinson's disease kinase LRRK2 was reported to target a subset of Rab proteins [181], yet an influence of Ser65-phosphoUb on LRRK2 was not tested.

More prevalent are the roles of Ser65-phosphoUb on mitochondria, where ubiquitin chain modifications are recognized by (phospho)Ub-binding proteins to initiate mitophagy, or targeted by DUBs or ubiquitin phosphatases to restrict mitophagy (see below). The most prominent and best-understood role of Ser65-phosphoUb on mitochondria is to recruit and activate Parkin.
A major conformation

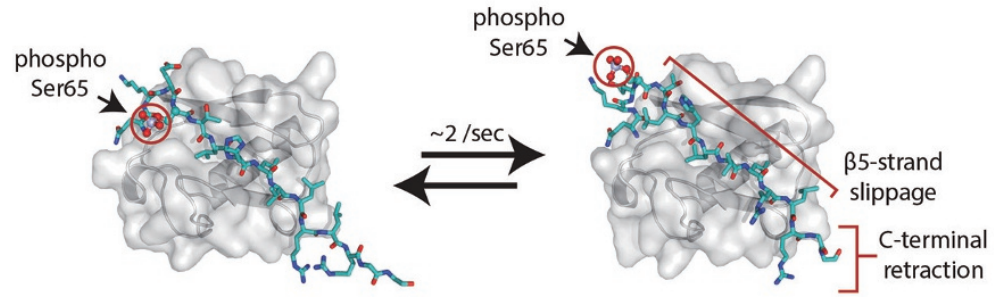

B

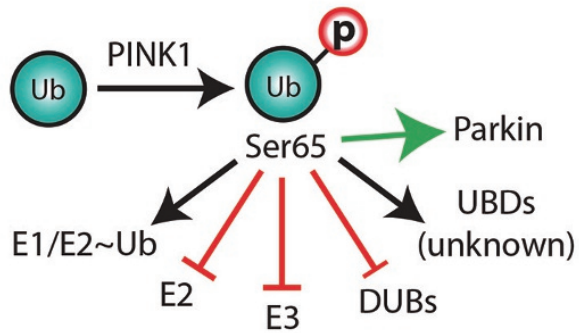

Figure 7 Structural and functional consequences of Ser65-phosphorylation of ubiquitin. (A) Ser65 phosphorylation generates a dynamic equilibrium between two ubiquitin conformations. A major conformation structurally resembles unmodified ubiquitin, but has altered electrostatic potential. The minor conformation has a retracted C-terminus induced by slippage of the $\beta 5$-strand. The images show the differing region in stick-representation embedded in the remaining ubiquitin core under a surface. The phosphorylated Ser65 is indicated. (B) Ser65-phosphorylation of ubiquitin has neutral, loss-of-function and gainof-function effects on components of the ubiquitin system. E1 and E2 charging is largely unaffected; however, E2 discharging and chain elongation mediated by a subset of E2 and E2/E3 chain assembly systems are inhibited. A substantial numbers of DUBs have reduced activity against Ser65-phosphoUb chains. Receptors recognizing Ser65-phosphoUb are unknown. Ser65-phosphoUb allosterically activates the E3 ligase Parkin and may activate kinase signaling towards Rab GTPases. 


\section{Regulation of Parkin activity}

Parkin recruitment to mitochondria leads to a rapid and striking increase in mitochondrial ubiquitination [91], however, Parkin also has roles outside of mitochondrial maintenance, e.g., in the restriction of Mycobacteria [98] and in cell signaling processes [182]. Parkin is primarily cytosolic, and its role in AR-JP has resulted in many studies of its activity and of its substrates. Like HOIP, Parkin belongs to the RBR family of E3 ligases that act via a covalent ubiquitin-bound intermediate [183]. Ubiquitin-charged E2 enzymes such as UBE2D or UBE2L3 are bound by the RING1 domain in Parkin, and transfer their ubiquitin not directly to a substrate Lys, but first onto a Cys residue in the RING2 domain (Figure 8). From there, substrates are modified; as mentioned above, Parkin predominantly generates Lys6-, Lys11- and to a lesser extent, Lys48- and Lys63-linked polyubiquitin, in chains of unstudied architecture [91, 92].

Importantly, structural and biochemical work has revealed that Parkin is autoinhibited in several ways (Figure 8A). An N-terminal Ubl domain in Parkin is autoinhibitory [184], but subsequent crystal structures of Parkin with and without the Ubl domain revealed a greater extent of autoinhibition [185-187]. Indeed, both the Ubl domain, and the repressor element of Parkin (REP) situated in the In-Between-RING (IBR) and RING2 domain linker, block the E2 binding site of the RING1 domain. Moreover, the catalytic Cys residue in the Parkin RING2 domain is inaccessible, as it is located at a tight, hydrophobic interface with the Unique Parkin Domain (UPD also referred to as RING0). Even if the E2 could bind to the RING1 domain, modeling suggests that the RING2 and E2 catalytic centers would be on opposite faces of the protein. These findings illustrated the need for significant conformational changes to activate Parkin [185-187].

Strikingly, the required conformational changes can be invoked by Ser65-phosphoUb. Robust ubiquitination by Parkin is observed in presence of PINK1, but purified Ser65-phosphoUb can bypass the requirement for PINK1. Curiously, Parkin cannot utilize Ser65-phosphoUb in chain assembly reactions [44, 91], but instead uses it as an allosteric regulator [172]. The molecular detail of this became clear from a crystal structure of Parkin in complex with Ser65-phosphoUb [188] (Figure 8B), which showed how a cryptic Ser65-phosphoUb-binding site not present in autoinhibited Parkin, is formed through straightening of a RING1 helix, inducing movement of the IBR domain. In the complex structure, Ser65-phosphoUb is cradled by the UPD, RING1 and IBR domains, explaining the nanomolar affinity [188] (Figure 8B). Conformational changes of the IBR domain has multiple effects, and destabilizes the REP-mediated RING1 repression, but more importantly, also leads to a release of the Ubl domain from the Parkin core [188-191]. Together, this enables binding of the charged E2 [172, 189]. The complex structure has not lost all of its autoinhibited character as the RING2:UPD interface continues to block access to the RING2 active site [91, 188]. Further conformational changes are hence required to fully unblock Parkin.

What such conformational changes might look like was revealed recently in a structure of the HOIP RBR domain module trapped in a catalytically active conformation bound to a ubiquitin-charged E2 enzyme [62] (Figure $8 \mathrm{C}$ ). In this structure, the RING2 domain is juxtaposed to the IBR domain to receive ubiquitin from the E2 active site. Excitingly, the IBR domain adopts an identical position with respect to the RING1 domain as observed in the Ser65-phosphoUb-bound Parkin structure [188], and the binding site for the E2-delivered 'donor' ubiquitin matches an additional ubiquitin-binding interface mapped in Parkin [184]. Intriguingly, in the HOIP structure, an additional, unmodified, ubiquitin occupies an identical binding site as Ser65-phosphoUb in Parkin (Figure 8C). While it is unclear whether HOIP undergoes similar conformational changes upon binding to this putative 'activator ubiquitin', the additional ubiquitin suggests that other RBR E3 ligases may be regulated by an 'activator ubiquitin', which in case of Parkin is an activator Ser65-phosphoUb. The RBR E3 ligase HHARI binds to and is activated by NEDD8 [192, 193], and it is tempting to speculate that NEDD8 serves a similar role as that of Ser65-phosphoUb in Parkin activation.

Finally, Ser65-phosphoUb-mediated release of the Ubl domain of Parkin not only unblocks the E2 binding site, but also enables its phosphorylation by PINK1, adding some much needed insight into the sequence of events leading to Parkin activation [188-191] (Figure 8C-8D). Parkin phosphorylation stabilizes it in its active form $[91,188]$, and we believe that the phosphorylated Ubl re-binds elsewhere on the Parkin core, covering hydrophobic surfaces exposed by the required conformational changes [188] (Figure 8D).

\section{Recognizing (phospho)ubiquitinated mitochondria}

In addition to recruiting and activating Parkin to significantly increase ubiquitination of mitochondria, Ser65-phosphoUb-containing chains might be the signal that distinguishes mitophagy from other forms of autophagy, as mitophagy depends on PINK1 [176]. A common characteristic of autophagy receptors is their ability to bind both ubiquitin as well as Ubl modifiers of 


\section{A Autoinhibited Parkin}

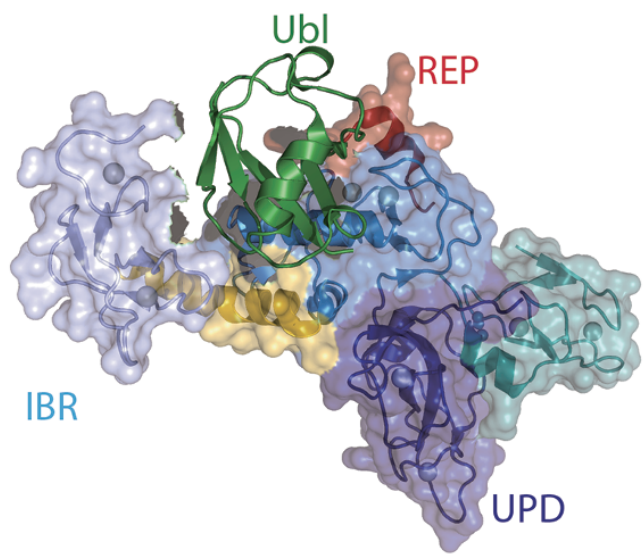

B Parkin Ser65-phosphoUb complex

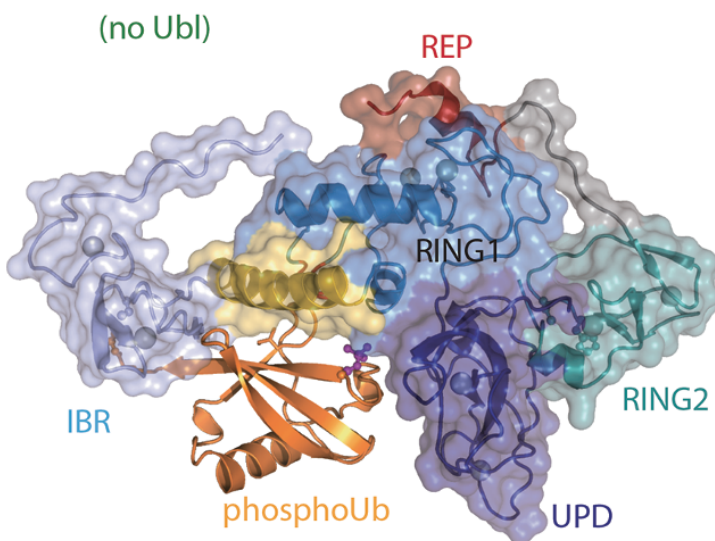

Sequence of events:

(1) phosphoUb binding

(2) Ubl release

(3) Ubl phosphorylation

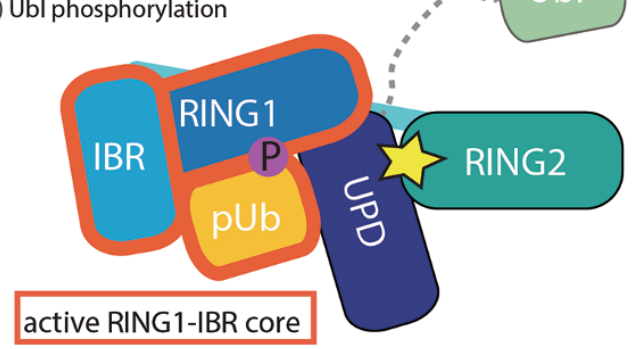

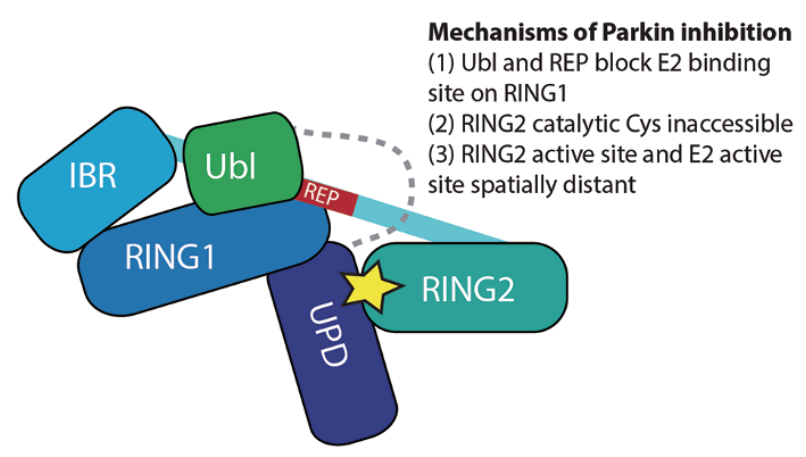

C Active HOIP RBR-E2 Ub-Ub complex

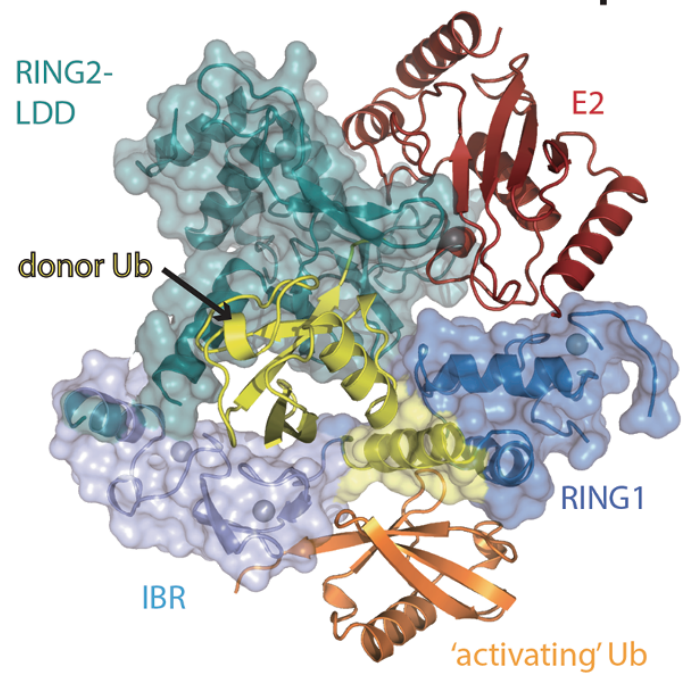

E2

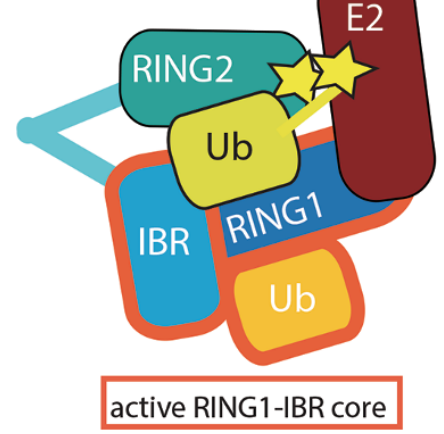

\section{Model for active Parkin}

Sequence of events:

(4) RING2/UPD opening \& REP release

(4a) phosphoUbl rebinding?

(5) E2 Ub binding

(6) transfer of Ub onto RING2

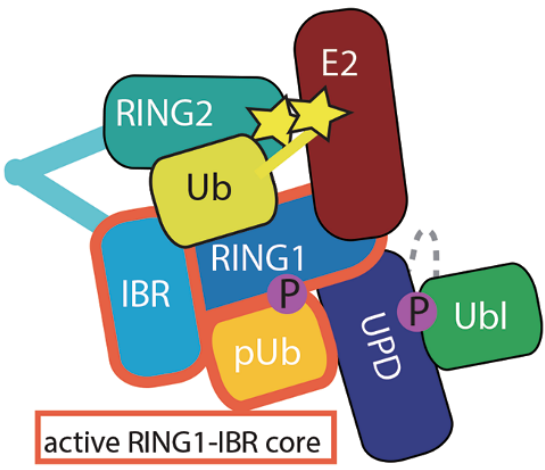


the Atg8-family that mark autophagosomal membranes [194].

Six autophagy receptors have been annotated, namely p62/SQSTM1, NBR1, NDP52, TAX1BP1, OPTN and TOLLIP [194]. Several studies have investigated which of these proteins are recruited to mitochondria in a PINK1- and Parkin-dependent manner [176, 177, 195]. The studies agree on important roles for NDP52 [176, 177] and OPTN [176, 177, 195] (Figure 6E). Mitophagy is strongly impaired when OPTN and NDP52 are deleted from cells, and lost completely when TAX1BP1 is also deleted in a triple knockout [176]. A role for p62/ SQSTM1 in mitophagy is more controversial. While the protein is recruited to damaged mitochondria [111, 176, $177,196,197]$, it does not appear to initiate mitophagy but instead promotes mitochondrial clustering [196, 197]. Autophagy receptors are expressed in a cell-type specific fashion, which may explain some of the discrepancies and observed redundancies, such as the recently reported role of p62/SQSTM1 in macrophage mitophagy after LPS stimulation [198].

Recruitment of autophagy adaptors to mitochondria depends on their ubiquitin binding properties and also on PINK1 [176, 177], suggesting that recognition of Ser65-phosphoUb is important. A preference for Ser65-phosphoUb binding could so far not be established in vitro, and additional factors or modifications on the receptors may be required $[176,177]$. Of particular importance is the protein kinase TBK1, which binds and phosphorylates many receptors, and modulates their adaptor functions [176, 177, 194, 199]. However, how mitophagy adaptors work on mitochondria remains unclear, and a bona-fide Ser65-phosphoUb receptor appears to still be missing. An even bigger question is whether Ser65-phosphoUb is involved in alternative forms of autophagy, such as xenophagy (the removal of pathogens from the cytosol [200]), which uses the identical receptors NDP52 and OPTN. As mentioned above, Parkin has been linked to bacterial restriction pathways [98], but it is unclear how it can be activated in other contexts.

\section{Antagonizing (or spatially restricting) mitophagy}

Bulk mitophagy as induced by depolarizing reagents is far from being physiological. In a real-life scenario, a mitochondrion, or a part of the continuously fusing and dividing mitochondrial network, is damaged, and this invokes mitophagy by the mechanisms described above, yet in a spatially restricted way. How an area of mitochondria can be tagged for disposal is unclear, but this could involve negative regulators of mitophagy. In principle, the mitophagy initiation signal, i.e., polyubiquitin comprising Ser65-phosphoUb molecules, could be antagonized by DUBs, or by ubiquitin phosphatases. While the identity of the latter is unknown, several DUBs have been proposed to directly regulate Parkin-mediated mitophagy initiation, including USP30 [93, 95, 96], USP35 [201], USP15 [202], USP8 [92] and Ataxin-3 [203] (Figure 6). The mechanisms of these enzymes are different: USP8 and Ataxin-3 were proposed to remove polyubiquitin from Parkin itself to stabilize the enzyme, and knockdown of USP8 prevents Parkin recruitment to mitochondria $[92,203]$. USP30 and USP15 were suggested to target Parkin substrates on mitochondria. USP8, USP15, and Ataxin-3 have many differing roles in cells [14], and are not dedicated to mitophagy/mitochondrial maintenance. The version of USP35 that was reported to antagonize mitophagy ('short-USP35') appears to lack a functional catalytic domain. Short-USP35 contains a mitochondrial targeting sequence (MTS) but is rapidly lost from mitochondria upon depolarization, and does not affect Parkin recruitment [201]. USP30 also contains an MTS and permanently resides on mitochondria to restrict mitophagy [93, 95, 96]. USP30 appears to be important in mitochondrial homeostasis as it actively removes ubiquitin from substrates such as TOM20 on healthy mitochondria (Figure 6A). This suggests that USP30 activity is switched off in mitophagy, which could occur via Parkin-mediated USP30 ubiquitination [95] (Figure 6D).

Interestingly, and consistent with their functions,

Figure 8 Insights into Parkin activation. (A) Structure of autoinhibited full-length Parkin (PDB ID: 4K95, [185]). Domains are colored in green (Ubl), dark-blue (UPD, also known as RING0), blue (RING1), light-blue (IBR), cyan (RING2) and red (REP). The mechanisms of Parkin inhibition are listed in the schematic figure. (B) Complex structure of Parkin bound to Ser65-phosphoUb. Coloring as in A with Ser65-phosphoUb in orange. Conformational changes in RING1 and IBR domain form the Ser65-phosphoUb-binding site. The Ubl domain was not included in the crystallized construct. (C) Structure of a HOIP RBR module bound to ubiquitin-charged E2 (yellow/red) and an extra, 'activator' ubiquitin. The structures in B and C are shown side-by-side to point out the identical active RING1-IBR module, indicated in the cartoon. Importantly, RING2 is juxtaposed to the E2 active site to receive ubiquitin. (D) The model of HOIP in C may indicate what active Parkin could look like. In this model, the RING2-UPD interface has been opened, and RING2 now sits atop RING1/IBR to receive ubiquitin. The hydrophobic surface on the UPD that was occupied by RING2, could be covered by rebinding of the phosphorylated Ubl domain [188]. 
Parkin and USP30 have a matching chain linkage preference, and make or cleave Lys6-linked chains preferentially [44, 93] (see above). How Ser65-phosphoUb-containing chains are cleaved is less clear, since many DUBs are significantly less active against polyubiquitin containing exclusively Ser65-phosphoUb [44, 45]. Finally, USP30 has also been shown to act as a negative regulator of Parkin-induced apoptotic cell death [96], suggesting that it serves as a critical modulator of not only mitophagy, but also in the maintenance of overall cellular health.

\section{Conclusions, lessons and future outlook}

Our understanding of the ubiquitin code has become much more sophisticated. Initially regarded as a defined means to degrade proteins, ubiquitination is now considered the most versatile protein modification system, impacting virtually any realm of life sciences. The discovery of many linkage-specific proteins and enzymes has alerted researchers that particular linkages must play distinct roles. The appreciation of the intricacies in the ubiquitin code has generated new methods and enabled routine checking of which linkage types are involved in particular systems. This led to new discoveries linking unstudied atypical chain types to new cellular processes, and has remarkably also started entirely new research areas, e.g., on ubiquitin phosphorylation. While information on several ubiquitin modifications is still scarce, the example of Met1-linked chains, or Ser65-phosphoUb signaling in mitophagy shows how combined efforts from many laboratories can lead to real progress in important research fields.

Chemical biology has enabled access to all ubiquitin linkage types as well as to new ubiquitin modifications, and will continue to provide essential tools to study the system as a whole. Yet, to study atypical chains or new modifications, the key was the identification of the enzymatic machineries that generate the modification, again, exemplified by LUBAC and PINK1. The recent rush in identifying new E3 ligases for atypical chains will likely prove essential to associate roles to these unstudied signals. It will also be essential to identify the enzymes regulating ubiquitin phosphorylation and acetylation for real progress to be made in these areas.

Indeed, most of the new insights into linkage-specific 'writers', 'readers' and 'erasers' of the ubiquitin code, originate in bottom-up biochemical approaches, followed by mechanistic studies by structural biology and biophysical techniques. The ubiquitin field is rich in mechanism, but somewhat lags behind in physiology. It is important that the biochemical facts and molecular details are incorporated in the physiological studies of the respective proteins; this is sometimes still missing from the current literature.

This review deals predominantly with the individual modifications of the ubiquitin molecule, and together, the distinctly modified ubiquitin moieties could be seen as the 'words' in the ubiquitin code. A further, even bigger and more challenging frontier awaits in studying the 'grammar' of the ubiquitin code. What is the architecture of polyubiquitin chains? How long can ubiquitin chains become in cells? How can we start to study mixed-linkage and branched chains, and do these polymers function differently? Are there underlying rules, akin to the hierarchy in modifications in the histone code, that exclude or enable combinations of modifications? How many combinations of modifications do we need to consider, and which ones can be detected in cells? Answering these questions requires the development of new technologies and methods, but addressing them will provide fundamental insights into ubiquitin and cellular biology.

While the ubiquitin system appears to be of mind-boggling complexity, the progress in the last decade is astounding and encourages us that the ubiquitin code can be cracked in the next few decades. We would hope that eventually, distinct modifications can be rapidly distinguished, architecture and interplay between modifications can be assessed, and downstream outcomes of the modifications can be predicted with confidence.

\section{Acknowledgments}

We apologize to those researchers whose work could not be cited. We would like to thank all past and present members of the lab for their shared excitement about the ubiquitin system, and Jonathan Pruneda, Rune Damgaard, Paul Elliott, Michal Simicek and Malte Gersch for comments on the manuscript. Work in the DK lab was funded by the Medical Research Council (U105192732), the European Research Council (309756), and the Lister Institute for Preventive Medicine.

\section{Competing Financial Interests}

DK is part of the DUB Alliance that includes Cancer Research Technology and FORMA Therapeutics, and is a consultant for FORMA Therapeutics.

\section{References}

1 Goldknopf IL, French MF, Musso R, Busch H. Presence of protein A24 in rat liver nucleosomes. Proc Natl Acad Sci USA 1977; 74:5492-5495.

2 Cohen P. The origins of protein phosphorylation. Nat Cell Biol 2002; 4:E127-E130.

3 Verdin E, Ott M. 50 years of protein acetylation: from gene regulation to epigenetics, metabolism and beyond. Nat Rev Mol Cell Biol 2015; 16:258-264.

4 Hershko A, Ciechanover A. The ubiquitin system. Annu Rev 
Biochem 1998; 67:425-479.

5 Clague MJ, Heride C, Urbé S. The demographics of the ubiquitin system. Trends Cell Biol 2015; 25:417-426.

6 Pickart CM. Mechanisms underlying ubiquitination. Annu Rev Biochem 2001; 70:503-533.

7 Schulman BA, Harper JW. Ubiquitin-like protein activation by E1 enzymes: the apex for downstream signaling pathways. Nat Rev Mol Cell Biol 2009; 10:319-331.

8 Ye Y, Rape M. Building ubiquitin chains: E2 enzymes at work. Nat Rev Mol Cell Biol 2009; 10:755-764.

9 Deshaies RJ, Joazeiro CAP. RING domain E3 ubiquitin ligases. Annu Rev Biochem 2009; 78:399-434.

10 Smit JJ, Sixma TK. RBR E3-ligases at work. EMBO Rep 2014; 15:142-154.

11 Rotin D, Kumar S. Physiological functions of the HECT family of ubiquitin ligases. Nat Rev Mol Cell Biol 2009; 10:398409.

12 Husnjak K, Dikic I. Ubiquitin-binding proteins: decoders of ubiquitin-mediated cellular functions. Annu Rev Biochem 2012; 81:291-322.

13 Komander D, Clague MJ, Urbé S. Breaking the chains: structure and function of the deubiquitinases. Nat Rev Mol Cell Biol 2009; 10:550-563.

14 Clague MJ, Barsukov I, Coulson JM, Liu H, Rigden DJ, Urbé S. Deubiquitylases from genes to organism. Physiol Rev 2013; 93:1289-1315.

15 Peng J, Schwartz D, Elias JE, et al. A proteomics approach to understanding protein ubiquitination. Nat Biotechnol 2003; 21:921-926.

16 Kim W, Bennett EJ, Huttlin EL, et al. Systematic and quantitative assessment of the ubiquitin-modified proteome. Mol Cell 2011; 44:325-340.

17 Wagner SA, Beli P, Weinert BT, et al. A proteome-wide, quantitative survey of in vivo ubiquitylation sites reveals widespread regulatory roles. Mol Cell Proteomics 2011; 10:M111.013284.

18 Kaiser SE, Riley BE, Shaler TA, et al. Protein standard absolute quantification (PSAQ) method for the measurement of cellular ubiquitin pools. Nat Methods 2011; 8:691-696.

19 Komander D, Rape M. The ubiquitin code. Annu Rev Biochem 2012; 81:203-229.

20 Jenuwein T, Allis CD. Translating the histone code. Science 2001; 293:1074-1080.

21 Xu P, Duong DM, Seyfried NT, et al. Quantitative proteomics reveals the function of unconventional ubiquitin chains in proteasomal degradation. Cell 2009; 137:133-145.

22 Dammer EB, Na CH, Xu P, et al. Polyubiquitin linkage profiles in three models of proteolytic stress suggest the etiology of Alzheimer disease. J Biol Chem 2011; 286:10457-10465.

23 Ziv I, Matiuhin Y, Kirkpatrick DS, et al. A perturbed ubiquitin landscape distinguishes between ubiquitin in trafficking and in proteolysis. Mol Cell Proteomics 2011; 10:M111.009753.

24 Chen ZJ, Sun LJ. Nonproteolytic functions of ubiquitin in cell signaling. Mol Cell 2009; 33:275-286.

25 Kulathu Y, Komander D. Atypical ubiquitylation - the unexplored world of polyubiquitin beyond Lys48 and Lys63 linkages. Nat Rev Mol Cell Biol 2012; 13:508-523.

26 Galisson F, Mahrouche L, Courcelles M, et al. A novel proteomics approach to identify SUMOylated proteins and their modification sites in human cells. Mol Cell Proteomics 2011; 10:M110.004796-M110.004796.

27 Lamoliatte F, Bonneil E, Durette C, et al. Targeted identification of SUMOylation sites in human proteins using affinity enrichment and paralog-specific reporter ions. Mol Cell Proteomics 2013; 12:2536-2550.

28 Hendriks IA, D’Souza RCJ, Yang B, Verlaan-de Vries M, Mann M, Vertegaal ACO. Uncovering global SUMOylation signaling networks in a site-specific manner. Nat Struct Mol Biol 2014; 21:927-936.

29 Choudhary C, Kumar C, Gnad F, et al. Lysine acetylation targets protein complexes and co-regulates major cellular functions. Science 2009; 325:834-840.

30 Lundby A, Lage K, Weinert BT, et al. Proteomic analysis of lysine acetylation sites in rat tissues reveals organ specificity and subcellular patterns. Cell Rep 2012; 2:419-431.

31 Ohtake F, Saeki Y, Sakamoto K, et al. Ubiquitin acetylation inhibits polyubiquitin chain elongation. EMBO Rep 2015; 16:192-201.

32 Weinert BT, Schölz C, Wagner SA, et al. Lysine succinylation is a frequently occurring modification in prokaryotes and eukaryotes and extensively overlaps with acetylation. Cell Rep 2013; 4:842-851.

33 Lee HJ, Na K, Kwon MS, Kim H, Kim KS, Paik YK. Quantitative analysis of phosphopeptides in search of the disease biomarker from the hepatocellular carcinoma specimen. Proteomics 2009; 9:3395-3408.

34 Zhou H, Di Palma S, Preisinger C, et al. Toward a comprehensive characterization of a human cancer cell phosphoproteome. J Proteome Res 2013; 12:260-271.

35 Lundby A, Secher A, Lage K, et al. Quantitative maps of protein phosphorylation sites across 14 different rat organs and tissues. Nat Commun 2012; 3:876.

36 Swaney DL, Beltrao P, Starita L, et al. Global analysis of phosphorylation and ubiquitylation cross-talk in protein degradation. Nat Methods 2013; 10:676-682.

37 Rikova K, Guo A, Zeng Q, et al. Global survey of phosphotyrosine signaling identifies oncogenic kinases in lung cancer. Cell 2007; 131:1190-1203.

38 Olsen JV, Blagoev B, Gnad F, et al. Global, in vivo, and site-specific phosphorylation dynamics in signaling networks. Cell 2006; 127:635-648.

39 Xia Z-P, Sun L, Chen X, et al. Direct activation of protein kinases by unanchored polyubiquitin chains. Nature 2009; 461:114-119.

40 Chen J, Chen ZJ. Regulation of NF- $\kappa$ B by ubiquitination. Curr Opin Immunol 2013; 25:4-12.

41 Rajsbaum R, García-Sastre A. Virology. Unanchored ubiquitin in virus uncoating. Science 2014; 346:427-428.

42 Fiesel FC, Ando M, Hudec R, et al. (Patho-)physiological relevance of PINK1-dependent ubiquitin phosphorylation. EMBO Rep 2015;16:1114-11130.

43 Lai YC, Kondapalli C, Lehneck R, et al. Phosphoproteomic screening identifies Rab GTPases as novel downstream targets of PINK1. EMBO J 2015; 34:2840-2861.

44 Wauer T, Swatek KN, Wagstaff JL, et al. Ubiquitin Ser65 phosphorylation affects ubiquitin structure, chain assembly and hydrolysis. EMBO J 2015; 34:307-325.

45 Swaney DL, Rodríguez-Mias RA, Villén J. Phosphorylation 
of ubiquitin at Ser65 affects its polymerization, targets, and proteome-wide turnover. EMBO Rep 2015; 16:1131-1144.

46 Hospenthal MK, Mevissen TET, Komander D. Deubiquitinase-based analysis of ubiquitin chain architecture using Ubiquitin Chain Restriction (UbiCRest). Nat Protoc 2015; 10:349-361.

47 Sims JJ, Scavone F, Cooper EM, et al. Polyubiquitin-sensor proteins reveal localization and linkage-type dependence of cellular ubiquitin signaling. Nat Methods 2012; 9:303-309.

48 van Wijk SJL, Fiskin E, Putyrski M, et al. Fluorescence-based sensors to monitor localization and functions of linear and K63-linked ubiquitin chains in cells. Mol Cell 2012; 47:797809.

49 Newton K, Matsumoto ML, Wertz IE, et al. Ubiquitin chain editing revealed by polyubiquitin linkage-specific antibodies. Cell 2008; 134:668-678.

50 Matsumoto ML, Wickliffe KE, Dong KC, et al. K11-linked polyubiquitination in cell cycle control revealed by a K11 linkage-specific antibody. Mol Cell 2010; 39:477-484.

51 Matsumoto ML, Dong KC, Yu C, et al. Engineering and structural characterization of a linear polyubiquitin-specific antibody. J Mol Biol 2012; 418:134-144.

52 Ordureau A, Münch C, Harper JW. Quantifying ubiquitin signaling. Mol Cell 2015; 58:660-676.

53 Kirisako T, Kamei K, Murata S, et al. A ubiquitin ligase complex assembles linear polyubiquitin chains. EMBO J 2006; 25:4877-4887.

54 Komander D, Reyes-Turcu F, Licchesi JDF, Odenwaelder P, Wilkinson KD, Barford D. Molecular discrimination of structurally equivalent Lys 63-linked and linear polyubiquitin chains. EMBO Rep 2009; 10:466-473.

55 Rahighi S, Ikeda F, Kawasaki M, et al. Specific recognition of linear ubiquitin chains by NEMO is important for NF-kappaB activation. Cell 2009; 136:1098-1109.

56 Tokunaga F, Sakata S-I, Saeki Y, et al. Involvement of linear polyubiquitylation of NEMO in NF-kappaB activation. Nat Cell Biol 2009; 11:123-132.

57 Haas TL, Emmerich CH, Gerlach B, et al. Recruitment of the linear ubiquitin chain assembly complex stabilizes the TNF-R1 signaling complex and is required for TNF-mediated gene induction. Mol Cell 2009; 36:831-844.

58 Gerlach B, Cordier SM, Schmukle AC, et al. Linear ubiquitination prevents inflammation and regulates immune signaling. Nature 2011; 471:591-596.

59 Smit JJ, Monteferrario D, Noordermeer SM, van Dijk WJ, van der Reijden BA, Sixma TK. The E3 ligase HOIP specifies linear ubiquitin chain assembly through its RING-IBRRING domain and the unique LDD extension. EMBO J 2012; 31:3833-3844.

60 Stieglitz B, Morris-Davies AC, Koliopoulos MG, Christodoulou E, Rittinger K. LUBAC synthesizes linear ubiquitin chains via a thioester intermediate. EMBO Rep 2012; 13:840846.

61 Stieglitz B, Rana RR, Koliopoulos MG, et al. Structural basis for ligase-specific conjugation of linear ubiquitin chains by HOIP. Nature 2013; 503:422-426.

62 Lechtenberg BC, Rajput A, Sanishvili R, et al. Structure of a HOIP/E2 ubiquitin complex reveals RBR E3 ligase mechanism and regulation. Nature 2016; 529:546-550.
63 Sato Y, Fujita H, Yoshikawa A, et al. Specific recognition of linear ubiquitin chains by the Npl4 zinc finger (NZF) domain of the HOIL-1L subunit of the linear ubiquitin chain assembly complex. Proc Natl Acad Sci USA 2011; 108:20520-20525.

64 Tokunaga F, Nishimasu H, Ishitani R, et al. Specific recognition of linear polyubiquitin by A20 zinc finger 7 is involved in NF- $\kappa \mathrm{B}$ regulation. EMBO J 2012; 31:3856-3870.

65 Verhelst K, Carpentier I, Kreike M, et al. A20 inhibits LUBAC-mediated NF- $\kappa \mathrm{B}$ activation by binding linear polyubiquitin chains via its zinc finger 7. EMBO J 2012; 31:38453855.

66 Ikeda F, Deribe YL, Skånland SS, et al. SHARPIN forms a linear ubiquitin ligase complex regulating NF- $\kappa$ B activity and apoptosis. Nature 2011; 471:637-641.

67 Tokunaga F, Nakagawa T, Nakahara M, et al. SHARPIN is a component of the NF- $\mathrm{BB}$-activating linear ubiquitin chain assembly complex. Nature 2011; 471:633-636.

68 Peltzer N, Rieser E, Taraborrelli L, et al. HOIP deficiency causes embryonic lethality by aberrant TNFR1-mediated endothelial cell death. Cell Rep 2014; 9:153-165.

69 Kumari S, Redouane Y, López-Mosqueda J, et al. Sharpin prevents skin inflammation by inhibiting TNFR1-induced keratinocyte apoptosis. 2014; 3:e03422.

70 Sasaki Y, Sano S, Nakahara M, et al. Defective immune responses in mice lacking LUBAC-mediated linear ubiquitination in B cells. EMBO J 2013; 32:2463-2476.

71 MacDuff DA, Reese TA, Kimmey JM, et al. Phenotypic complementation of genetic immunodeficiency by chronic herpesvirus infection. eLife 2015; 4:e04494.

72 Park Y, Jin H-S, Lopez J, et al. SHARPIN controls regulatory $\mathrm{T}$ cells by negatively modulating the $\mathrm{T}$ cell antigen receptor complex. Nat Immunol 2016; 17:286-296.

73 Boisson B, Laplantine E, Dobbs K, et al. Human HOIP and LUBAC deficiency underlies autoinflammation, immunodeficiency, amylopectinosis, and lymphangiectasia. J Exp Med 2015; 212:939-951.

74 Boisson B, Laplantine E, Prando C, et al. Immunodeficiency, autoinflammation and amylopectinosis in humans with inherited HOIL-1 and LUBAC deficiency. Nat Immunol 2012; 13:1178-1186.

75 Walczak H, Iwai K, Dikic I. Generation and physiological roles of linear ubiquitin chains. BMC Biol 2012; 10:23.

76 Iwai K, Fujita H, Sasaki Y. Linear ubiquitin chains: NF-kB signaling, cell death and beyond. Nat Rev Mol Cell Biol 2014; 15:503-508.

77 Sato Y, Goto E, Shibata Y, et al. Structures of CYLD USP with Met1- or Lys63-linked diubiquitin reveal mechanisms for dual specificity. Nat Struct Mol Biol 2015; 22:222-229.

78 Keusekotten K, Elliott PR, Glockner L, et al. OTULIN antagonizes LUBAC signaling by specifically hydrolyzing Met1linked polyubiquitin. Cell 2013; 153:1312-1326.

79 Rivkin E, Almeida SM, Ceccarelli DF, et al. The linear ubiquitin-specific deubiquitinase gumby regulates angiogenesis. Nature 2013; 498:318-324.

80 Fiil BK, Damgaard RB, Wagner SA, et al. OTULIN restricts Met1-linked ubiquitination to control innate immune signaling. Mol Cell 2013; 50:818-830.

81 Elliott PR, Nielsen SV, Marco-Casanova P, et al. Molecular basis and regulation of OTULIN-LUBAC interaction. $\mathrm{Mol}$ 
Cell 2014; 54:335-348.

82 Schaeffer V, Akutsu M, Olma MH, Gomes LC, Kawasaki M, Dikic I. Binding of OTULIN to the PUB domain of HOIP controls NF-kB signaling. Mol Cell 2014; 54:349-361.

83 Takiuchi T, Nakagawa T, Tamiya H, et al. Suppression of LUBAC-mediated linear ubiquitination by a specific interaction between LUBAC and the deubiquitinases CYLD and OTULIN. Genes Cells 2014; 19:254-272.

84 Draber P, Kupka S, Reichert M, et al. LUBAC-recruited CYLD and A20 regulate gene activation and cell death by exerting opposing effects on linear ubiquitin in signaling complexes. Cell Rep 2015; 13:2258-2272.

85 Mevissen TET, Hospenthal MK, Geurink PP, et al. OTU Deubiquitinases reveal mechanisms of linkage specificity and enable ubiquitin chain restriction analysis. Cell 2013; 154:169184.

86 De A, Dainichi T, Rathinam CV, Ghosh S. The deubiquitinase activity of A20 is dispensable for NF- $\mathrm{B}$ signaling. EMBO Rep 2014; 15:775-783.

87 Wertz IE, Newton K, Seshasayee D, et al. Phosphorylation and linear ubiquitin direct A20 inhibition of inflammation. Nature 2015; 528:370-375.

88 Elia AEH, Boardman AP, Wang DC, et al. Quantitative proteomic atlas of ubiquitination and acetylation in the DNA damage response. Mol Cell 2015; 59:867-881.

89 Wu-Baer F, Lagrazon K, Yuan W, Baer R. The BRCA1/ BARD1 heterodimer assembles polyubiquitin chains through an unconventional linkage involving lysine residue K6 of ubiquitin. J Biol Chem 2003; 278:34743-34746.

90 Morris JR, Solomon E. BRCA1 : BARD1 induces the formation of conjugated ubiquitin structures, dependent on K6 of ubiquitin, in cells during DNA replication and repair. Hum Mol Genet 2004; 13:807-817.

91 Ordureau A, Sarraf SA, Duda DM, et al. Quantitative proteomics reveal a feedforward mechanism for mitochondrial PARKIN translocation and ubiquitin chain synthesis. Mol Cell 2014; 56:360-375.

92 Durcan TM, Tang MY, Pérusse JR, et al. USP8 regulates mitophagy by removing K6-linked ubiquitin conjugates from parkin. EMBO J 2014; 33:2473-2491.

93 Cunningham CN, Baughman JM, Phu L, et al. USP30 and parkin homeostatically regulate atypical ubiquitin chains on mitochondria. Nat Cell Biol 2015; 17:160-169.

94 Ordureau A, Heo J-M, Duda DM, et al. Defining roles of PARKIN and ubiquitin phosphorylation by PINK1 in mitochondrial quality control using a ubiquitin replacement strategy. Proc Natl Acad Sci USA 2015; 112:6637-6642.

95 Bingol B, Tea JS, Phu L, et al. The mitochondrial deubiquitinase USP30 opposes parkin-mediated mitophagy. Nature 2014; 510:370-375.

96 Liang J-R, Martinez A, Lane JD, Mayor U, Clague MJ, Urbé S. USP30 deubiquitylates mitochondrial Parkin substrates and restricts apoptotic cell death. EMBO Rep 2015; 16:618-627.

97 Faesen AC, Luna-Vargas MPA, Geurink PP, et al. The differential modulation of USP activity by internal regulatory domains, interactors and eight ubiquitin chain types. Chem Biol 2011; 18:1550-1561.

98 Manzanillo PS, Ayres JS, Watson RO, et al. The ubiquitin ligase parkin mediates resistance to intracellular pathogens.
Nature 2013; 501:512-516.

99 Hospenthal MK, Freund SMV, Komander D. Assembly, analysis and architecture of atypical ubiquitin chains. Nat Struct Mol Biol 2013; 20:555-565.

100 Lin DY-W, Diao J, Zhou D, Chen J. Biochemical and structural studies of a HECT-like ubiquitin ligase from Escherichia coli O157:H7. J Biol Chem 2011; 286:441-449.

101 Bremm A, Komander D. Emerging roles for Lys11-linked polyubiquitin in cellular regulation. Trends Biochem Sci 2011; 36:355-363.

102 Wickliffe KE, Williamson A, Meyer H-J, Kelly A, Rape M. K11-linked ubiquitin chains as novel regulators of cell division. Trends Cell Biol 2011; 21:656-663.

103 Grice GL, Lobb IT, Weekes MP, Gygi SP, Antrobus R, Nathan JA. The proteasome distinguishes between heterotypic and homotypic lysine-11-linked polyubiquitin chains. Cell Rep 2015; 12:545-553.

104 Meyer H-J, Rape M. Enhanced protein degradation by branched ubiquitin chains. Cell 2014; 157:910-921.

105 Min M, Mevissen TET, De Luca M, Komander D, Lindon C. Efficient APC/C substrate degradation in cells undergoing mitotic exit depends on K11 ubiquitin linkages. Mol Biol Cell 2015; 26:4325-4332.

$106 \mathrm{Hu} \mathrm{H}$, Brittain GC, Chang J-H, et al. OTUD7B controls non-canonical NF- $\kappa \mathrm{B}$ activation through deubiquitination of TRAF3. Nature 2013; 494:371-374.

$107 \mathrm{Hu} \mathrm{H}$, Wang H, Xiao Y, et al. Otud7b facilitates T cell activation and inflammatory responses by regulating Zap70 ubiquitination. $J$ Exp Med 2016; 213:399-414.

108 Pareja F, Ferraro DA, Rubin C, et al. Deubiquitination of EGFR by Cezanne-1 contributes to cancer progression. Oncogene 2012; 31:4599-4608.

109 Bremm A, Moniz S, Mader J, Rocha S, Komander D. Cezanne (OTUD7B) regulates HIF-1 $\alpha$ homeostasis in a proteasome-independent manner. EMBO Rep 2014; 15:1268-1277.

110 Moniz S, Bandarra D, Biddlestone J, et al. Cezanne regulates E2F1-dependent HIF2 $\alpha$ expression. J Cell Sci 2015; 128:3082-3093.

111 Geisler S, Holmström KM, Skujat D, et al. PINK1/Parkin-mediated mitophagy is dependent on VDAC1 and p62/SQSTM1. Nat Cell Biol 2010; 12:119-131.

112 Gatti M, Pinato S, Maiolica A, et al. RNF168 Promotes noncanonical K27 ubiquitination to signal DNA damage. Cell Rep 2015; 10:226-238.

113 Liu Z, Chen P, Gao H, et al. Ubiquitylation of autophagy receptor Optineurin by HACE1 activates selective autophagy for tumor suppression. Cancer Cell 2014; 26:106-120.

114 Palicharla VR, Maddika S. HACE1 mediated K27 ubiquitin linkage leads to YB-1 protein secretion. Cell Signal 2015; 27:2355-2362.

115 You J, Pickart CM. A HECT domain E3 enzyme assembles novel polyubiquitin chains. J Biol Chem 2001; 276:1987119878.

116 Michel MA, Elliott PR, Swatek KN, et al. Assembly and specific recognition of k29- and k33-linked polyubiquitin. $\mathrm{Mol}$ Cell 2015; 58:95-109.

117 Kristariyanto YA, Abdul Rehman SA, Campbell DG, et al. K29-selective ubiquitin binding domain reveals structural basis of specificity and heterotypic nature of k29 polyubiquitin. 
Mol Cell 2015; 58:83-94.

118 Besche HC, Sha Z, Kukushkin NV, et al. Autoubiquitination of the $26 \mathrm{~S}$ proteasome on Rpn13 regulates breakdown of ubiquitin conjugates. EMBO J 2014; 33:1159-1176.

119 Crosas B, Hanna J, Kirkpatrick DS, et al. Ubiquitin chains are remodeled at the proteasome by opposing ubiquitin ligase and deubiquitinating activities. Cell 2006; 127:1401-1413.

120 Virdee S, Ye Y, Nguyen DP, Komander D, Chin JW. Engineered diubiquitin synthesis reveals Lys29-isopeptide specificity of an OTU deubiquitinase. Nat Chem Biol 2010; 6:750757.

121 Licchesi JDF, Mieszczanek J, Mevissen TET, et al. An ankyrin-repeat ubiquitin-binding domain determines TRABID's specificity for atypical ubiquitin chains. Nat Struct Mol Biol 2012; 19:62-71.

122 Tran H, Hamada F, Schwarz-Romond T, Bienz M. Trabid, a new positive regulator of Wnt-induced transcription with preference for binding and cleaving K63-linked ubiquitin chains. Genes Dev 2008; 22:528-542.

123 Jin J, Xie X, Xiao Y, et al. Epigenetic regulation of the expression of I112 and II23 and autoimmune inflammation by the deubiquitinase Trabid. Nat Immunol 2016; 17:259-268.

124 Kristariyanto YA, Choi S-Y, Rehman SAA, et al. Assembly and structure of Lys33-linked polyubiquitin reveals distinct conformations. Biochem J 2015; 467:345-352.

125 Kim JB, Kim SY, Kim BM, et al. Identification of a novel anti-apoptotic E3 ubiquitin ligase that ubiquitinates antagonists of inhibitor of apoptosis proteins SMAC, HtrA2, and ARTS. $J$ Biol Chem 2013; 288:12014-12021.

126 Yuan W-C, Lee Y-R, Lin S-Y, et al. K33-linked polyubiquitination of Coronin 7 by Cul3-KLHL20 ubiquitin E3 ligase regulates protein trafficking. Mol Cell 2014; 54:586-600.

127 Thrower JS, Hoffman L, Rechsteiner M, Pickart CM. Recognition of the polyubiquitin proteolytic signal. EMBO J 2000; 19:94-102.

128 Sakata E, Bohn S, Mihalache O, et al. Localization of the proteasomal ubiquitin receptors Rpn10 and Rpn13 by electron cryomicroscopy. Proc Natl Acad Sci USA 2012; 109:14791484.

129 Lander GC, Estrin E, Matyskiela ME, Bashore C, Nogales E, Martin A. Complete subunit architecture of the proteasome regulatory particle. Nature 2012; 482:186-191.

130 Matyskiela ME, Lander GC, Martin A. Conformational switching of the $26 \mathrm{~S}$ proteasome enables substrate degradation. Nat Struct Mol Biol 2013; 20:781-788.

131 Kirkpatrick DS, Hathaway NA, Hanna J, et al. Quantitative analysis of in vitro ubiquitinated cyclin B1 reveals complex chain topology. Nat Cell Biol 2006; 8:700-710.

132 Flick K, Ouni I, Wohlschlegel JA, et al. Proteolysis-independent regulation of the transcription factor Met4 by a single Lys 48-linked ubiquitin chain. Nat Cell Biol 2004; 6:634-641.

133 Flick K, Raasi S, Zhang H, Yen JL, Kaiser P. A ubiquitin-interacting motif protects polyubiquitinated Met4 from degradation by the 26S proteasome. Nat Cell Biol 2006; 8:509-515.

$134 \mathrm{Lu}$ Y, Lee B-H, King RW, Finley D, Kirschner MW. Substrate degradation by the proteasome: a single-molecule kinetic analysis. Science 2015; 348:1250834.

135 Kanayama A, Seth RB, Sun L, et al. TAB2 and TAB3 activate the NF-kappaB pathway through binding to polyubiquitin chains. Mol Cell 2004; 15:535-548.

136 Kulathu Y, Akutsu M, Bremm A, Hofmann K, Komander D. Two-sided ubiquitin binding explains specificity of the TAB2 NZF domain. Nat Struct Mol Biol 2009; 16:1328-1330.

137 Sato Y, Yoshikawa A, Yamashita M, Yamagata A, Fukai S. Structural basis for specific recognition of Lys 63-linked polyubiquitin chains by NZF domains of TAB2 and TAB3. EMBO $J$ 2009; 28:3903-3909.

138 Ivins FJ, Montgomery MG, Smith SJM, Morris-Davies AC, Taylor IA, Rittinger K. NEMO oligomerization and its ubiquitin-binding properties. Biochem J 2009; 421:243-251.

139 Emmerich $\mathrm{CH}$, Ordureau A, Strickson S, et al. Activation of the canonical IKK complex by K63/M1-linked hybrid ubiquitin chains. Proc Natl Acad Sci USA 2013; 110:15247-15252.

140 Laplantine E, Fontan E, Chiaravalli J, et al. NEMO specifically recognizes K63-linked poly-ubiquitin chains through a new bipartite ubiquitin-binding domain. EMBO J 2009; 28:28852895.

141 Hadian K, Griesbach RA, Dornauer S, et al. NEMO interaction with linear and K63 ubiquitin chains contributes to NF-\{kappa\}B activation. J Biol Chem 2011; 286:2610726117.

142 Boname JM, Thomas M, Stagg HR, Xu P, Peng J, Lehner PJ. Efficient internalization of MHC I requires lysine-11 and lysine-63 mixed linkage polyubiquitin chains. Traffic 2010; 11:210-220.

143 Goto E, Yamanaka Y, Ishikawa A, et al. Contribution of Lysine 11-linked ubiquitination to MIR2-mediated major histocompatibility complex Class I internalization. J Biol Chem 2010; 285:35311-35319.

144 Kim HT, Kim KP, Lledias F, et al. Certain pairs of ubiquitin-conjugating enzymes (E2s) and ubiquitin-protein ligases (E3s) synthesize nondegradable forked ubiquitin chains containing all possible isopeptide linkages. J Biol Chem 2007; 282:17375-17386.

145 Kim HT, Kim KP, Uchiki T, Gygi SP, Goldberg AL. S5a promotes protein degradation by blocking synthesis of nondegradable forked ubiquitin chains. EMBO J 2009; 28:18671877.

$146 \mathrm{Xu}$ P, Peng J. Characterization of polyubiquitin chain structure by middle-down mass spectrometry. Anal Chem 2008; 80:3438-3444.

147 Valkevich EM, Sanchez NA, Ge Y, Strieter ER. Middle-down mass spectrometry enables characterization of branched ubiquitin chains. Biochemistry 2014; 53:4979-4989.

148 Finley D. Recognition and processing of ubiquitin-protein conjugates by the proteasome. Annu Rev Biochem 2009; 78:477-513.

149 Pierce NW, Kleiger G, Shan S-O, Deshaies RJ. Detection of sequential polyubiquitylation on a millisecond timescale. $\mathrm{Na}$ ture 2009; 462:615-619.

150 Ye Y, Blaser G, Horrocks MH, et al. Ubiquitin chain conformation regulates recognition and activity of interacting proteins. Nature 2012; 492:266-270.

151 Schaefer JB, Morgan DO. Protein-linked ubiquitin chain structure restricts activity of deubiquitinating enzymes. $J$ Biol Chem 2011; 286:45186-45196.

152 Streich FC, Lima CD. Structural and functional insights to ubiquitin-like protein conjugation. Annu Rev Biophys 2014; 
43:357-379

153 Flotho A, Melchior F. Sumoylation: a regulatory protein modification in health and disease. Annu Rev Biochem 2013; 82:357-385.

154 Sriramachandran AM, Dohmen RJ. SUMO-targeted ubiquitin ligases. Biochim Biophys Acta 2014; 1843:75-85.

155 Singh RK, Zerath S, Kleifeld O, Scheffner M, Glickman MH, Fushman D. Recognition and cleavage of related to ubiquitin 1 (Rub1) and Rub1-ubiquitin chains by components of the ubiquitin-proteasome system. Mol Cell Proteomics 2012; 11:1595-1611.

156 Hjerpe R, Thomas Y, Chen J, et al. Changes in the ratio of free NEDD8 to ubiquitin triggers NEDDylation by ubiquitin enzymes. Biochem J 2012; 441:927-936.

157 Olsen JV, Mann M. Status of large-scale analysis of post-translational modifications by mass spectrometry. $\mathrm{Mol}$ Cell Proteomics 2013; 12:3444-3452.

158 Neumann H, Peak-Chew SY, Chin JW. Genetically encoding $\mathrm{N}$ (epsilon)-acetyllysine in recombinant proteins. Nat Chem Biol 2008; 4:232-234.

159 Wickliffe KE, Lorenz S, Wemmer DE, Kuriyan J, Rape M. The mechanism of linkage-specific ubiquitin chain elongation by a single-subunit E2. Cell 2011; 144:769-781.

160 Lorenz S, Bhattacharyya M, Feiler C, Rape M, Kuriyan J. Crystal structure of a Ube2S-ubiquitin conjugate. PLoS One 2016; 11:e0147550.

161 Youle RJ, Narendra DP. Mechanisms of mitophagy. Nat Rev Mol Cell Biol 2011; 12:9-14.

162 Sugiura A, McLelland G-L, Fon EA, McBride HM. A new pathway for mitochondrial quality control: mitochondrial-derived vesicles. EMBO J 2014; 33:2142-2156.

163 Jin SM, Lazarou M, Wang C, Kane LA, Narendra DP, Youle RJ. Mitochondrial membrane potential regulates PINK1 import and proteolytic destabilization by PARL. J Cell Biol 2010; 191:933-942.

164 Yamano K, Youle RJ. PINK1 is degraded through the N-end rule pathway. Autophagy 2013; 9:1758-1769.

165 Narendra DP, Jin SM, Tanaka A, et al. PINK1 is selectively stabilized on impaired mitochondria to activate Parkin. PLoS Biol 2010; 8:e1000298.

166 Narendra D, Tanaka A, Suen D-F, Youle RJ. Parkin is recruited selectively to impaired mitochondria and promotes their autophagy. J Cell Biol 2008; 183:795-803.

167 Chen Y, Dorn GW. PINK1-phosphorylated mitofusin 2 is a Parkin receptor for culling damaged mitochondria. Science 2013; 340:471-475.

168 Wang X, Winter D, Ashrafi G, et al. PINK1 and Parkin target Miro for phosphorylation and degradation to arrest mitochondrial motility. Cell 2011; 147:893-906.

169 Kondapalli C, Kazlauskaite A, Zhang N, et al. PINK1 is activated by mitochondrial membrane potential depolarization and stimulates Parkin E3 ligase activity by phosphorylating Serine 65. Open Biol 2012; 2:120080.

170 Shiba-Fukushima K, Imai Y, Yoshida S, et al. PINK1-mediated phosphorylation of the Parkin ubiquitin-like domain primes mitochondrial translocation of Parkin and regulates mitophagy. Sci Rep 2012; 2:1002.

171 Kane LA, Lazarou M, Fogel AI, et al. PINK1 phosphorylates ubiquitin to activate Parkin E3 ubiquitin ligase activity. J Cell
Biol 2014; 205:143-153.

172 Koyano F, Okatsu K, Kosako H, et al. Ubiquitin is phosphorylated by PINK1 to activate parkin. Nature 2014; 510:162166.

173 Kazlauskaite A, Kondapalli C, Gourlay R, et al. Parkin is activated by PINK1-dependent phosphorylation of ubiquitin at Ser65. Biochem J 2014; 460:127-139.

174 Shiba-Fukushima K, Arano T, Matsumoto G, et al. Phosphorylation of mitochondrial polyubiquitin by PINK1 promotes Parkin mitochondrial tethering. PLoS Genet 2014; 10:e1004861.

175 Okatsu K, Koyano F, Kimura M, et al. Phosphorylated ubiquitin chain is the genuine Parkin receptor. J Cell Biol 2015; 209:111-128.

176 Lazarou M, Sliter DA, Kane LA, et al. The ubiquitin kinase PINK1 recruits autophagy receptors to induce mitophagy. $\mathrm{Na}$ ture 2015; 524:309-314.

177 Heo J-M, Ordureau A, Paulo JA, Rinehart J, Harper JW. The PINK1-PARKIN mitochondrial ubiquitylation pathway drives a program of OPTN/NDP52 recruitment and TBK1 activation to promote mitophagy. Mol Cell 2015; 60:7-20.

178 Woodroof HI, Pogson JH, Begley M, et al. Discovery of catalytically active orthologues of the Parkinson's disease kinase PINK1: analysis of substrate specificity and impact of mutations. Open Biology 2011; 1:110012.

179 Perica T, Chothia C. Ubiquitin - molecular mechanisms for recognition of different structures. Curr Opin Struct Biol 2010; 20:367-376.

180 Lange OF, Lakomek N-A, Farès C, et al. Recognition dynamics up to microseconds revealed from an RDC-derived ubiquitin ensemble in solution. Science 2008; 320:1471-1475.

181 Steger M, Tonelli F, Ito G, et al. Phosphoproteomics reveals that Parkinson's disease kinase LRRK2 regulates a subset of Rab GTPases. eLife 2016; 5:e12813.

182 Müller-Rischart AK, Pilsl A, Beaudette P, et al. The E3 ligase parkin maintains mitochondrial integrity by increasing linear ubiquitination of NEMO. Mol Cell 2013; 49:908-921.

183 Wenzel DM, Lissounov A, Brzovic PS, Klevit RE. UBCH7 reactivity profile reveals parkin and HHARI to be RING/ HECT hybrids. Nature 2011; 474:105-108.

184 Chaugule VK, Burchell L, Barber KR, et al. Autoregulation of Parkin activity through its ubiquitin-like domain. EMBO J 2011; 30:2853-2867.

185 Trempe J-F, Sauvé V, Grenier K, et al. Structure of parkin reveals mechanisms for ubiquitin ligase activation. Science 2013; 340:1451-1455.

186 Wauer T, Komander D. Structure of the human Parkin ligase domain in an autoinhibited state. EMBO J 2013; 32:20992112.

187 Riley BE, Lougheed JC, Callaway K, et al. Structure and function of Parkin E3 ubiquitin ligase reveals aspects of RING and HECT ligases. Nat Commun 2013; 4:1982.

188 Wauer T, Simicek M, Schubert A, Komander D. Mechanism of phospho-ubiquitin-induced PARKIN activation. Nature 2015; 524:370-374.

189 Sauvé V, Lilov A, Seirafi M, et al. A Ubl/ubiquitin switch in the activation of Parkin. EMBO J 2015; 34:2492-2505.

190 Kumar A, Aguirre JD, Condos TE, et al. Disruption of the autoinhibited state primes the E3 ligase parkin for activation and 
catalysis. EMBO J 2015; 34:2506-2521.

191 Kazlauskaite A, Martinez-Torres RJ, Wilkie S, et al. Binding to serine 65-phosphorylated ubiquitin primes Parkin for optimal PINK1-dependent phosphorylation and activation. EMBO Rep 2015; 16:939-954.

192 Duda DM, Olszewski JL, Schuermann JP, et al. Structure of HHARI, a RING-IBR-RING ubiquitin ligase: Autoinhibition of an ariadne-family E3 and insights into ligation mechanism. Structure 2013; 21:1030-1041.

193 Kelsall IR, Duda DM, Olszewski JL, et al. TRIAD1 and HHARI bind to and are activated by distinct neddylated Cullin-RING ligase complexes. EMBO J 2013; 32:2848-2860.

194 Zaffagnini G, Martens S. Mechanisms of selective autophagy. J Mol Biol 2016; pii:S0022-2836(16)00109-1.

195 Wong YC, Holzbaur ELF. Optineurin is an autophagy receptor for damaged mitochondria in parkin-mediated mitophagy that is disrupted by an ALS-linked mutation. Proc Natl Acad Sci USA 2014; 111:E4439-E4448.

196 Narendra D, Kane LA, Hauser DN, Fearnley IM, Youle RJ. p62/SQSTM1 is required for Parkin-induced mitochondrial clustering but not mitophagy; VDAC1 is dispensable for both. Autophagy 2010; 6:1090-1106.

197 Okatsu K, Saisho K, Shimanuki M, et al. p62/SQSTM1 cooperates with Parkin for perinuclear clustering of depolarized mitochondria. Genes Cells 2010; 15:887-900.

198 Zhong Z, Umemura A, Sanchez-Lopez E, et al. NF-кB restricts inflammasome activation via elimination of damaged mitochondria. Cell 2016; 164:896-910.

199 Wild P, Farhan H, McEwan DG, et al. Phosphorylation of the autophagy receptor optineurin restricts Salmonella growth. Science 2011; 333:228-233.

200 Randow F, Youle RJ. Self and nonself: how autophagy targets mitochondria and bacteria. Cell Host Microbe 2014; 15:403411.

201 Wang Y, Serricchio M, Jauregui M, et al. Deubiquitinating enzymes regulate PARK2-mediated mitophagy. Autophagy 2015; 11:595-606.

202 Cornelissen T, Haddad D, Wauters F, et al. The deubiquitinase USP15 antagonizes Parkin-mediated mitochondrial ubiquitination and mitophagy. Hum Mol Genet 2014; 23:5227-5242.

203 Durcan TM, Kontogiannea M, Bedard N, Wing SS, Fon EA. Ataxin-3 deubiquitination is coupled to Parkin ubiquitination via E2 ubiquitin-conjugating enzyme. J Biol Chem 2012; 287:531-541.

\section{(c) (i)}

This work is licensed under a Creative Commons Attribution 4.0 Unported License. The images or other third party material in this article are included in the article's Creative Commons license, unless indicated otherwise in the credit line; if the material is not included under the Creative Commons license, users will need to obtain permission from the license holder to reproduce the material. To view a copy of this license, visit http:// creativecommons.org/licenses/by/4.0/ 